\title{
Application of a Hybrid ARIMA-LSTM Model Based on The SPEI For Drought Forecasting
}

\section{Dehe Xu}

North China University of Water Resources and Electric Power

Qi Zhang ( $\nabla 95300576 @ q q . c o m$ )

North China University of Water Resources and Electric Power

\section{Yan Ding}

North China University of Water Resources and Electric Power

De Zhang

State Key Laboratory of Geo-Information Engineering

\section{Research Article}

Keywords: Drought forecasting, SPEI, ARIMA-SVR, LS-SVR, ARIMA-LSTM

Posted Date: May 10th, 2021

DOl: https://doi.org/10.21203/rs.3.rs-301080/v1

License: (c) (i) This work is licensed under a Creative Commons Attribution 4.0 International License.

Read Full License

Version of Record: A version of this preprint was published at Environmental Science and Pollution Research on August 17th, 2021. See the published version at https://doi.org/10.1007/s11356-021-15325Z. 

Application of a hybrid ARIMA-LSTM model based on the SPEI for drought forecasting integrated moving average (ARIMA) model and a long short-term memory (LSTM) model to improve the accuracy of short-term drought prediction. Taking China as an example, this paper compares and analyzes the prediction accuracy of six drought prediction models, ARIMA, support vector regression (SVR), LSTM, ARIMA-SVR,

15 least square-SVR (LS-SVR) and ARIMA-LSTM, for SPEI. The performance of all the 16 models was compared using measures of persistence, such as the Nash-Sutcliffe 17 efficiency (NSE) and so on. The results show that all three hybrid models (ARIMA-

18 SVR, LS-SVR and ARIMA-LSTM) had higher prediction accuracy than the single 19 model. (ARIMA, SVR and LSTM), for a given lead time, at different scales. the NSEs 20 of the hybrid ARIMA-SVR, LS-SVR and ARIMA-LSTM models for the predicted

\footnotetext{
${ }^{1}$ Corresponding author (Qi Zhang). Tel.: +86 13253659813; E-mail address: 895300576@qq.com
} 
21 SPEI1 are $0.043,0.168$ and 0.368 , respectively, and the NSEs of SPEI24 is $0.781,0.543$

22 and 0.93 , respectively. This finding indicates that when the lead time remains

23 unchanged, the prediction accuracy of the hybrid ARIMA-SVR, LS-SVR and ARIMA-

24 LSTM models for the SPEI at various scales is gradually improved with increasing time

25 scale, and the prediction accuracy of the model with a one-month lead time is higher

26 than that of the model with a two-month lead time. In addition, the ARIMA-LSTM

27 model has the highest prediction accuracy at the 6-, 12-, and 24-month scales, indicating

28 that the model is more suitable for the forecasting of long-term drought in China. 


\section{Introduction}

33 From a global perspective, drought is one of the most serious natural disasters in

34 the world, with the widest impact and incurring greatest economic losses (Tian et al.

35 2018). In recent years, global warming, excessive carbon emissions and other issues

36 have led to the continued increase of agricultural droughts, which seriously threatens

37 China's domestic and global food production, and accurate assessment, monitoring

38 and analysis of drought has been a hot topic of domestic and foreign scholars.

39 Therefore, quantitative research on drought is conducive to improving drought

40 monitoring, strengthening the development of drought prediction, and identifying

41 strategies. Generally, easy-to-calculate drought indicators are used to monitor and

42 evaluate the drought intensity, duration and disaster area (Zhang et al. 2019). Due to

43 the wide application range and variety of drought indicators and the different

44 understanding of drought in different majors and disciplines, a variety of drought

45 indicators have been proposed (Yao et al. 2018a).

46 Common drought indices include the meteorological-related Palmer drought

47 severity index (PDSI) (Paulo et al. 2012; Vicente-Serrano et al. 2015; Dai et al. 2004),

48 SPI (Mckee et al. 1993) and SPEI (Vicente-Serrano et al. 2010), the soil water

49 content-related soil moisture anomaly index (SMAI) (Gao et al. 2016) and

50 evapotranspiration deficit index (ETDI) (Narasimhan and Srinivasan 2005) and the

51 hydrological-related Palmer hydrological drought index (PHDI) (Tatli and Türkeş

52 2011) and standardized water-level index (SWI) (Bhuiyan et al. 2006). In addition, the 
remote sensing-based normalized differential vegetation index (NDVI) (Gu et al.

54 2007) is also frequently used. Both the PDSI and SPEI require temperature and precipitation data, and the SMAI and ETDI are more complicated to calculate than the SPI. The SWI only considers groundwater, the NDVI requires long-term data, and satellite data have no long-term history. SPI is multi-scale and computationally simple because rainfall is the only input data (Chen and Sun 2015; Wang et al. 2015, 2016). The SPEI is based on the SPI but adds a temperature component that allows the SPEI to account for the effect of temperature on the development of drought through basic water balance calculations (Soh et al. 2018; Yao et al. 2018b). Li et al. (2020) elucidated drought characteristics of China during 1980-2015 using two commonly used meteorological drought indices: SPI and SPEI. Given the fact that potential evapotranspiration increases in a warming climate, Li et al. (2020) show that SPEI may be more suitable than SPI in monitoring drought under climate change. Therefore, the SPEI were chosen to monitor drought in this study. forecasting(Adamowski 2008; Mossad and Alazba 2015; Karthika et al. 2017; RafieiSardooi et al. 2018; Fung et al. 2019b). There are many models used to forecast drought, such as the ARIMA model (Mishra and Desai 2005, 2006; Mishra et al.

71 2007; Han et al. 2010), which is the most widely adopted stochastic approach. Tian et 72 al. (2016) used the ARIMA model to predict the agricultural drought in the 73 Guanzhong Plain, mainly on the time series of the VTCI drought monitoring results; 
74 the results showed that the absolute error of the AR(1) model was lower than that of

75 the SARIMA model, both in frequency distribution and statistical results. It shows

76 that the ARIMA model can better predict the type and extent of drought and can be

77 used for drought prediction in the plains. However, the author did not conduct the

78 analysis and prediction from multiple time scales, so the results obtained were limited

79 and the model was not necessarily applicable to all time scales. Mossad and Alazba

80 (2015) developed several ARIMA models for drought forecasting using multiscale

81 SPEI in hyperarid climates, and showed that ARIMA models can be very useful tools

82 for drought forecasting that can help water resource managers and planners to take

83 precautions considering the severity of drought in advance. However, stochastic

84 models are linear models with limited ability to predict nonlinear data; therefore,

85 when the time scale is large, ARIMA models cannot perform well at all scales. In

86 recent years, ANN models have been used by researchers to improve the prediction

87 accuracy of nonlinear data, as traditional data-driven models do not predict nonlinear

88 data well(Ochoa-Rivera 2008; Marj and Meijerink 2011; Sigaroodi et al. 2013;

89 Kousari et al. 2017; Seibert et al. 2017). ANNs have been used as drought prediction

90 tools in many studies (Belayneh and Adamowski 2012; Deo and Şahin 2015;

91 Belayneh et al. 2016; Borji et al. 2016; Chen et al. 2017; Seibert et al. 2017) and have

92 achieved good results. There are many kinds of ANNs, including recurrent neural

93 networks (RNNs) and LSTM networks (Poornima and Pushpalatha 2019). RNNs and

94 traditional ANNs have been compared in terms of time series model prediction, and it 
has been found that RNNs achieve a higher prediction accuracy than traditional ANNs. RNNs have shown significant effects on the forecasting of data sequences. However, when the sequence length is too large, there is a dramatic increase in the training time of the RNN. Based on the above problems, the LSTM network (Hochreiter 1997) is proposed, which improves the hidden layer of the RNN, expands the memory function of the network, enables the model to obtain more persistent information, and slows down the information decay rate (Tran Anh et al. 2019). LSTM is a kind of deep learning method and has made great progress in time series prediction (Zhang et al. 2018; Jeong and Park 2019; Qi et al. 2019; Tran Anh et al. 2019). However, it is difficult to use LSTM architecture in short-term time series prediction or with meteorological drought indices. Poornima et al. (2019) used LSTM to predict the multitime scale SPI and SPEI and found that the model could better process real-time nonlinear data and achieve good the long-term prediction. However, because the prediction accuracy is not high or cannot improve the short-term prediction accuracy, a variety of hybrid models are used to predict the drought index. Modaresi et al. (2018) used four data-driven models to forecast the monthly inflow to the $\mathrm{K}$ dam, and a cross-validation approach was used to determine the optimal values of the model parameters. The results show that the LS-SVR model performs best under nonlinear conditions and the ANN performs best under linear conditions. Xu et al. (2020) used the ARIMA model and hybrid ARIMA-SVR model, for predicting the SPEI with a lead time of one month. By applying the mean absolute error (MAE), root 
117 metrics, it was found that the ARIMA-SVR model was superior with the trend in

118 improving accuracy when the timescale of the SPI increased. Therefore this paper

119 uses ARIMA-LSTM, LS-SVR and ARIMA-SVR models for comparison of

120 prediction accuracy.

121 The objective of this paper is to propose a hybrid model with the advantages of a

122 linear and nonlinear models to improve the short-term prediction accuracy. The aim of

123 this paper is to use the 6 models to predict the multiscale SPEI computed from 613

124 sites in China, and to explore the prediction accuracy of different models at each scale

125 with the help of evaluation and validation metrics. Because the kriging interpolation

126 method is commonly used in drought index analyses (Manatsa et al. 2008; Karavitis et

127 al. 2012; Afzali et al. 2016; Jain and Flannigan 2017; Cai et al. 2019), this paper will

128 describe the use of the improved kriging interpolation method, empirical Bayesian

129 kriging (EBK), in ArcGIS to conduct a visual analysis of SPEI12 observed values and

130 fitted values of the three hybrid models from 2014 and 2015. The performance of all

131 the models is compared using measures of persistence, namely, the mean squared

132 error (MSE), NSE, RMSE and MAE.

134 2. Material and methods

1352.1 Study area and data 
much diversified topography (Fig 1). Considering the multi-year average temperature,

138 precipitation and moisture conditions, China is divided into seven natural sub-regions

139 (Yao et al. 2018b), which include the temperate and warm-temperate desert of

140 northwest China (sub-region 1), the temperate grassland of Inner Mongolia (sub-

141 region 2), the temperate humid and sub-humid northeast China (sub-region 3), the

142 warm-temperate humid and sub-humid north China (sub-region 4), the subtropical

143 humid central and south China (sub-region 5), the Qinghai-Tibetan Plateau (sub-

144 region 6), and the tropic humid south China (sub-region 7). The original data were

145 derived from the National Meteorological Information Center (http://data.cma.cn).

$146 \quad 2.2$ Methods

$147 \quad 2.2 .1 \mathrm{SPEI}$

148 In 2009, the World Meteorological Organization (WMO) recommended the

149 SPEI as the main meteorological drought indices, which countries can use to monitor

150 and track drought conditions (Hayes et al. 1996). By defining the SPEI as widely used

151 indices, the WMO provides guidance to countries seeking to establish early warning

152 levels for droughts. The probability density function (PDF) that has been used for the

153 SPEI is the log-logistic function, as suggested by Mckee et al. (1993) and Vicente-

154 Serrano et al. (2010), respectively. Since both Yao et al. (2018b) and Li et al. (2020)

155 used the SPEI to monitor the drought situation in China, the log-logistic PDFs are

156 available for meteorological sites in China. 
158 Commission on Irrigation and Drainage (ICID) as the standard procedure for

159

160

161

162

$163 \mathrm{P}$ is the probability of a definite $\mathrm{D}$ value:

164

165 For $\mathrm{P} \leq 0.5$,

166

167

168 For $\mathrm{P}>0.5$,

171

173 the SPEI is shown in Table 1.

174

175

176

177

178 the model ordering process can be found in detail in Xu et al. (2020). ARIMA models

Where $c_{0}=2.515517, c_{1}=0.802853, c_{2}=0.010328, d_{1}=1.432788, d_{2}=0.189269$, $d_{3}=0.001308$. The classification of dry and wet spells resulting from the values of 2.2.2 ARIMA modeling steps

ARIMA is a linear model that has been widely used in drought prediction in recent years (Kisi et al. 2015; Choubin and Malekian 2017). The formula for ARIMA and 
179

were developed based on the Box and Jenkins approach. The formula for a general non-seasonal ARIMA model can be abbreviated as follows(Xu et al. 2020):

$$
\begin{gathered}
Z_{t}=\frac{\theta(B) \alpha_{t}}{\phi(B) \nabla^{d}} \\
\phi B=\left(1-\phi_{t} B-\phi_{2} B^{2}-\cdots \phi_{p} B^{p}\right)
\end{gathered}
$$

And

$$
\theta B=\left(1-\theta_{1} B-\theta_{2} B^{2}-\cdots \theta_{q} B^{q}\right)
$$

where $Z_{t}$ is the observed time series and $\mathrm{B}$ is a back shift operator. $\phi B$ and $\theta B$ are polynomials of order $p$ and $q$, respectively. $\nabla^{d}$ describes the differencing operation to data series to make the data series stationary and $d$ is the number of regular differencing.

In this paper, ACF and PACF (Fig.6) are used to order the model, and AIC, BIC and HQIC parametrics were combined to select the optimal model parameters.

Finally, $80 \%$ of the data were selected as the training set and $20 \%$ as the test set using a cross-validation approach. The results of the SPEI with one month of lead time are shown in Fig. 8 and Fig. 9, respectively, and the results of the one-to-two-month lead time are shown in Table 4 and Table 5.

\subsubsection{LS-SVR model}

Support vector machine regression is a support vector machine algorithm used to solve regression problems, which is an extension and application of support vector machines to regression estimation problems. If we extend the conclusions obtained by 
200 the support vector machine for classification problems to the regression function, it

201 becomes support vector regression, which is commonly used for time series

202 prediction, nonlinear modeling and prediction, and optimization control(Modaresi et

203 al. 2018). Details and formulas about the SVR can be seen in the article by Xu et al.

204 (2020).

205 LS-SVMR, or least-squares support vector regression, was proposed by J.A.K

206 Suykens (Suykens et al. 2002) as an improvement to the SVM to facilitate its solution.

207 Compared with the standard SVM, LS-SVMR replaces the inequality constraint in the

208 SVM with an equation constraint, and the solution process becomes a set of equation

209 equations, which avoids the time-consuming quadratic planning problem of solution

210 and speeds up the solution process relatively fast. Details and formulas about the SVR

211 can be seen in the article by Suykens et al. (2002).

212 The LS-SVR modeling steps are as follows:

213 First of all, the data is standardized processing, because this article was used radial

214 basis function (RBF), so to calculate the distance of two points in high-dimensional

215 space, it is necessary to standardize the data first, in order to avoid the effect of the

216 scale on the calculation distance. Secondly, set the parameters sigma and gama, where

217 sigma represents the degree of dispersion of the point distribution in high-dimensional

218 space, gama balances the two items of the target function, and this paper uses cross-

219 validation to give the appropriate sigma value and gama value. 
LSTM is a variant of the RNN network. Compared to traditional RNNs, its hidden layer module structure has been greatly redesigned. Although RNNs can effectively

224 handle nonlinear time series, there are still two problems: (1) Due to gradient vanishing and gradient explosion, RNNs cannot handle time series with excessive delays; (2) training the RNN model requires a predetermined delay window length, but it is difficult to automatically obtain the optimal value of this parameter. Thus, the LSTM model emerged. The LSTM model replaces the hidden layer of RNN cells with LSTM cells to achieve long-term memory. The special design of the LSTM model makes it able to learn long-term dependencies and is particularly suitable for time 231 series analyses. specific structure is shown in Fig. 2. For the sake of understanding, memory modules

234 are often compared to computer memory. The memory module consists of a storage

235 unit and three computing components. The three computing components are called

236 input gates, output gates, and forget gates, which control the reading, writing, and 237 resetting of memory cell data, respectively. As seen from the figure, LSTM is a four-

238 layer structure, and each structure is connected to and interacts with the others. The

239 yellow part of the figure shows the various activation functions inside the memory

240 module, and the red part represents the basic operation of the vector, for example,

241 vector addition and product operations. The arrows represent the direction in which 
242 the vector is transmitted in the network. The confluence of two solid lines in the

243 figure indicates the vector combination of two parts. A solid line is divided into

244 multiple lines to indicate that the vector is copied and then flows to different parts of

245 the network.

246 The calculation of the LSTM hidden layer is shown in Fig. 3, and its forward

247 calculation method can be expressed as:

$$
\begin{gathered}
i_{t}=\sigma\left(W_{i} x_{t}+U_{i} h_{t-1}+b_{i}\right) \\
f_{t}=\sigma\left(W_{f} x_{t}+U_{f} h_{t-1}+b_{f}\right) \\
C_{t}=f_{t} \otimes C_{t-1}+i_{t} \otimes \widetilde{C}_{t} \\
\widetilde{C}_{t}=\tanh \left(W_{c} x_{t}+U_{c} h_{t-1}+b_{c}\right) \\
o_{t}=\sigma\left(W_{o} x_{t}+U_{o} h_{t-1}+b_{o}\right) \\
h_{t}=o_{t} \otimes \tanh \left(C_{t}\right)
\end{gathered}
$$

254 where $i, f, C$ and $o$ denote the input gate, forget gate, the cell state vector and 255 output gate, respectively. $W$ denotes the matrix of weights, $b$ denotes the bias

256 vectors, and $U$ denotes the matrix of weights to the hidden; $\sigma$ and tanh are sigmoid 257 and hyperbolic tangent activation functions, respectively. The elementwise

258 multiplication of two vectors is denoted by $\otimes$.

259 In this paper, multitime scale SPEI values were used in the prediction because 260 the data are a time series related to the previous sequence value. Since the number of 261 hidden layers determines the model's fitting ability, to prevent overfitting, this paper 
proposes an early stopping technique, which stops training when the loss function no

263 longer drops. The loss function is defined below:

$$
\text { LOSS } \left.=\sum_{i=1}^{N}\left(y_{i}-\widehat{y}_{l}\right)^{2}\right)
$$

265 where $y_{i}$ is the measured value at time $i$ and $\widehat{y}_{l}$ is the predicted value at time $i$.

266 The LSTM modeling steps are as follows:

267 1) Preprocessing of the input data

268 The original time series should not be directly input into the model as input data.

269 To obtain better training results, the original time series needs to be preprocessed.

270 Usually, the data processed by a neural network model are normalized data because

271 the training effect of the model is affected by the amount of original data. The

272 normalized data limit the data to a range of $[-1,1]$, which can eliminate the impact on

273 the neural network due to differences in the dimensions of each dataset, facilitate the

274 analysis of data, and improve the training speed of the model.

275 2) Model construction

276 The LSTM network in this paper was built based on the Keras framework of the

277 Python 3.7 platform. Therefore, before modeling the training data, the methods and

278 parameters need to be configured in the LSTM model based on the Keras framework.

279 The hidden layer of the LSTM in this paper was composed of 20 storage units, the

280 number of iterations was 300 , the batch size was set to 1 , the activation function was

281 set to ReLU, the loss function was the MSE, and the optimizer was SGD. However,

282 the number of hidden layers directly determines the quality of the model's fitting 
283 ability. To avoid overfitting, the MSE is adopted to describe and select the

284 performance of the model corresponding to the number of hidden layers of the LSTM

285 network.

286 3) Fitting model and parameter tuning

287 The golden section method was used in this paper to select the optimal number of

288 hidden neurons. The golden section method involves first finding the ideal number of

289 hidden layer nodes in the interval [a, b], expanding the search interval according to

290 the golden section principle, that is, obtaining the interval $[b, c]$ (where $b=0.619^{*}(c a)+$

291 a), and searching for the best value in $[b, c]$. The batch size is the number of samples

292 of model weight updates. The weight was updated after each sample. Therefore, the

293 batch size was set to 1 , and the process is called random gradient descent. Common

294 activation functions are sigmoid, tanh, and ReLU. Because the time-based back

295 propagation algorithm was used for network training, the sigmoid function is prone to

296 gradient vanishing during backpropagation and was unable to complete deep network

297 training, the SGD obtained by ReLU converged much faster than that obtained by the

298 sigmoid and tanh activation functions, so ReLU was used as the activation function in

299 this paper. The number of iterations was set as high as possible. The early stopping

300 method was used in this article to prevent overfitting. Since the number of training

301 cycles made the process very time-consuming, the loss function is set to check the

302 performance of the model on the training and verification dataset. Once overlearning

303 starts, training stops, so early stopping was used to suppress overfitting; that is, after 
305 increases, the training was stopped, and the weight after stopping was taken as the

306 final parameter of the network.

307 4) Training output and anti-normalization

308 The training data were programmed into the network training model, and when

309 the number of iterations exceeded the set threshold, the training ended. The output

310 value of the network was not the final forecast result of the time series. The actual

311 output of the model could be obtained by performing reverse normalization on the

312 output value of the network. Usually, this method is called inverse normalization.

\section{$314 \quad 2.2 .5$ Hybrid ARIMA-LSTM model}

315 In the time series forecasting problem, the characteristics of the linear model and

316 the nonlinear model determine that the former can only recognize the linear pattern of

317 the time series, and the latter has the advantage of being able to mine the nonlinear

318 relationship of the time series. A large number of experiments and applications have

319 shown that using a single model can have a good effect when dealing with a single

320 time series of components (Choubin et al. 2016; Soh et al. 2018; Fung et al. 2019b, a).

321 However, in the face of complex problems, a single model had certain limitations.

322 Because the time series of the research object contains linear components and

323 nonlinear components, a single linear model or a single nonlinear model is not

324 suitable for use. 
325 A hybrid model was established using the advantages of both the ARIMA and

326 LSTM models, as same as hybrid ARIMA-SVR model (Xu et al. 2020a). The

327 formulas are as follows:

328

$$
Y_{t}=L_{t}+N_{t}
$$

329 where $Y_{t}$ is a combination of linear $\left(L_{t}\right)$ and nonlinear parts $\left(N_{t}\right)$.

330 The modeling flow chart is shown in Fig. 4.

$331 \quad 2.2 .5$ Evaluation indices

332 In this study, MSE, RMSE, NSE (Nash JE 1970) and MAE were used as indicators

333 of model evaluation by the following formulas(Belayneh et al. 2016; Deo and Şahin

334 2016):

$$
\begin{aligned}
M S E & =\frac{S S E}{N} \\
R M S E & =\sqrt{\frac{S S E}{N}}
\end{aligned}
$$

337 where the SSE is the sum of squared errors and N is the number of samples. The SSE

338 is given by:

$$
S S E=\sum_{i=1}^{N}\left(y_{i}-\widehat{y}_{l}\right)^{2}
$$

340 with the variables already having been defined.

$$
N S E=1-\frac{\sum_{i=1}^{N}\left(y_{i}-\widehat{y}_{l}\right)^{2}}{\sum_{i=1}^{N}\left(y_{i}-\bar{y}\right)^{2}}
$$

342 where

$$
\bar{y}=\frac{\sum_{i=1}^{N} y_{i}}{N}
$$


344 where $y_{i}$ is the observed value at time $i(i=1, \cdots, N), \bar{y}$ is the mean value taken

345 over $\mathrm{N}$ and $\mathrm{N}$ is the total data size of $y_{i},(i=1, \cdots, N)$ and $\widehat{y}_{l}$ is the forecasted value 346 at time $i$.

$$
M A E=\frac{1}{N} \sum_{i=1}^{N}\left|\widehat{y}_{l}-y_{i}\right|
$$

3. Results and discussion

\subsection{Calculation results of SPEI}

The matplotlib visualization library in Python 3.6 was used to visualize the

Figs. 5. As can be seen from Fig 5, the SPEI of 7 sub-regions in China is on the rise, especially the severity of the drought in sub-region 2, 4, and 6. But on the 12-month scale, the drought in regions 1,2 , and 6 is relatively serious in recent 5 year.

\subsection{ARIMA, SVR and LSTM model predicts results}

In this paper, the ADF test was conducted in the 7 regions of China, and the $\mathrm{P}$ that SPEI1, SPEI3, SPEI6, SPEI12 and SPEI24 are stationary time series. The optimal ARIMA parameters of the multiscale SPEI of the 7 sub-regions are selected through the AIC, BIC and HQIC (as shown in Table 3). As the drought situation in subregion 1 is more serious than that in other regions, the sub-region 1 is selected as an 
example for time series prediction. The prediction results in other regions can be seen

366 in Figs. 10. The results of the one-month lead time of the SPEI are shown in Fig. 8,

367 and the results of the one-to-two-month lead time are shown in Table 4 and Table 5.

368 As seen from Figs. 8, the prediction accuracy of the ARIMA model is the highest for

369 SPEI24 and the lowest for SPEI1. As the time scale increases, the prediction accuracy

370 gradually increases because the ARIMA model is essentially an overall linear

371 autoregressive model that predicts a trend that tends to stabilize as the test set grows

372 (Yurekli et al. 2005; Hu et al. 2007).

373 In this paper, monthly precipitation and temperature data from 613 national

374 meteorological stations from 1980 to 2019 are used to conduct LSTM and SVR

375 modeling with multitime scale SPEI values. Due to the large number of regions, only

376 the same region by the ARIMA model were selected for results presentation for

377 comparative study, and the same dataset predicted by the ARIMA model after cross-

378 validation was also selected. The parameters of the LSTM model are shown in Fig.7.

379 It can be seen from Fig. 8 that the prediction results of the SVR and LSTM models

380 has the lowest accuracy at SPEI1 and the highest accuracy at SPEI24, and the

381 prediction accuracy increases gradually with increasing time scale. And we found that

382 the prediction accuracy of SVR is higher than that of ARIMA model at SPEI1 and

383 SPEI3, but it is lower than that of ARIMA model at SPEI6, SPEI12 and SPEI24. This

384 shows that SVR is more suitable for long-term drought prediction than ARIMA

385 model. LSTM has recently been used in many applications, including drought 
prediction (Poornima and Pushpalatha 2019), weather prediction (Salman et al. 2018), and water table depth prediction (Zhang et al. 2018), and has achieved good results because it can describe nonlinear relationships. Fig. 7 show that the box diagram of the LSTM model predicting the number of hidden layer neurons of the SPEI at various scales shows that with the increase in the number of hidden layers, the MSE gradually decreases, indicating that the more hidden layers there are, the higher the prediction accuracy of the LSTM. However, to prevent overfitting, this paper adopts a regularization method, namely, the early stopping method (described in section 2.2.3). At present, most studies on LSTM prediction models do not explain the problems of LSTM hidden layers and the prediction accuracy or the relationship between them.

3.3 Hybrid ARIMA-SVR, LS-SVR and ARIMA-LSTM model prediction results The flow of the ARIMA, SVR and LSTM modeling processes were described sections 2.2.2, 2.2.3 and 2.2.4, respectively, and the SPEI values were predicted separately. This paper proposes the ARIMA-LSTM hybrid model. Specifically, the

401 ARIMA model was used to extract the linear features of the multitime scale SPEI.

402 The residual between the predicted value and actual value of the ARIMA model were 403 entered into the LSTM model for prediction, and the advantages of the deep learning 404 method for time series were used to extract the nonlinear features. The linear part and 405 nonlinear part were combined to obtain the prediction results of the hybrid ARIMA406 LSTM model, and the flow chart is shown in Fig. 4. The results are shown in Fig. 9. 
At the same time, four kinds of experimental evaluation indices were selected in this paper. The hybrid ARIMA-LSTM model proposed in this paper was compared with the ARIMA, SVR, LSTM, ARIMA-SVR and LS-SVR model. The feasibility of the hybrid ARIMA-LSTM model for SPEI forecasting was verified. The effectiveness is

411 shown in Table 4 and Table 5.

Figs. 8-9 show that the predicted accuracy of the SPEI of the hybrid ARIMA-

413 LSTM model at all scales is higher than that of the single ARIMA, SVR, LSTM and hybrid ARIMA-SVR, LS-SVR model. With the increase in the time scale, the precision of the hybrid ARIMA-LSTM model increases gradually because the

416 precision values of the single models increase gradually. Xu et al. (2020) combined the ARIMA model and SVR model and predicted multiscale SPI by using a hybrid model and a single ARIMA model. This paper is similar in that the hybrid linear

419 model and nonlinear model is used for multiscale prediction, and the difference is that 420 this paper is mainly aimed at improving the short-term prediction accuracy and 421 avoiding model overfitting by using a regularization method, so it is more applicable. It can be seen from Fig. 5 that the severe drought in the past 10 years in 7 sub-

423 regions of China occurred in 2014 and 2015. The EBK method was used to

424 demonstrate the spatial distribution of the model prediction results of SPEI12 in China 425 in 2014-2015, as shown in Fig. 10. It was found that the prediction accuracy of the 426 three hybrid models (ARIMA-SVR, LS-SVR and ARIMA-LSTM) is similar in spatial 427 distribution. It can be seen from Fig.9 and Table 5, the prediction accuracy of SPEI6, 
SPEI12 and SPEI24 of the ARIMA-LSTM model is highest, indicating that the ARIMA-LSTM model is suitable for long-term drought prediction.

\section{Conclusions}

In this paper, the six prediction models (ARIMA, SVR, LSTM, ARIMA-SVR, LS-SVR, ARIMA-LSTM) are selected based on their advantages of predicting multiscale SPEI in 7 sub-regions of China. Four evaluation indices were selected to evaluate the prediction accuracy of the three models in the 1-2-month lead time, and the spatial distribution of the prediction results of the three models was displayed in combination with the EBK method.

Based on the key statistical parameters, the performance of the three hybrid models (ARIMA-SVR, LS-SVR, ARIMA-LSTM) increases gradually with increasing time scale. And the Prediction accuracy of the three hybrid models is higher than that of the three single models (ARIMA, SVR, LSTM). According to Table 4, we can see that the prediction accuracy of hybrid ARIMA-LSTM model is the highest at each timescale, especially at 6-,12-, and 24 months. When the lead time was one month, the prediction accuracy of hybrid ARIMA-LSTM model was lowest at SPEI1 and highest at SPI24 and SPEI24. When the prediction area remains unchanged, we find that the prediction accuracy of the three hybrid models decreases at all time scales with the increases in the prediction advance period; that is, the prediction accuracy of the model with a one-month lead time is higher than that of the model with a two-month 
449 lead time. From the spatial distribution diagram of the prediction model, the hybrid

450 ARIMA-LSTM model has higher prediction accuracy than the other models,

451 indicating that the hybrid model can contribute to improving the short-term prediction

452 accuracy and more suitable of long-term scale drought in China of meteorological

453 drought.

454

455 Declarations

456

- Ethics approval and consent to particaipate

$457 \quad$ Not applicable

458 - Consent for publication

$459 \quad$ Not applicable

460 - Availability of data and materials

461 The datasets used and/or analysed during the current study are available from the

462 corresponding author on reasonable request

463 - Authors Contributions

464 Q.Z conceived and designed the study. Y.D and D.Z collected data. Q.Z and D.X

465 conducted the simulations and performed the analyses, also provided critical

466 insights on the results interpretation. Q.Z wrote the initial draft of the paper, with

$467 \quad$ substantial contributions from all authors.

- Funding

469 This research was supported by State Key Laboratory of GeoInformation 
The authors declare that they have no known competing financial interests or personal relationships that could have appeared to influence the work reported in this paper.

\section{References:}

Adamowski JF (2008) Development of a short-term river flood forecasting method for snowmelt driven floods based on wavelet and cross-wavelet analysis. J Hydrol 353:247-266. https://doi.org/10.1016/j.jhydrol.2008.02.013

Afzali A, Keshtkar H, Pakzad S, et al (2016) Spatio-Temporal Analysis of Drought Severity Using Drought Indices and Deterministic and Geostatistical Methods (Case Study: Zayandehroud River Basin). Desert 21:165-172.

Aiguo D, Kevin ET, Taotao Q (2004) A Global Dataset of Palmer Drought Severity https://doi.org/10.22059/jdesert.2016.60325 Index for 1870 - 2002 : Relationship with Soil Moisture and Effects of Surface Warming. J Hydrometeorol 5:1117-1130. https://doi.org/10.1016/j.molcel.2017.04.015 
492

493

494

495

496

497

498

499

500

501

502

503

504

505

506

507

508

509

510

511

Belayneh A, Adamowski J, Khalil B, Quilty J (2016) Coupling machine learning methods with wavelet transforms and the bootstrap and boosting ensemble approaches for drought prediction. Atmos Res 172-173:37-47. https://doi.org/10.1016/j.atmosres.2015.12.017

Bhuiyan C, Singh RP, Kogan FN (2006) Monitoring drought dynamics in the Aravalli region (India) using different indices based on ground and remote sensing data. Int J Appl Earth Obs Geoinf 8:289-302. https://doi.org/10.1016/j.jag.2006.03.002

Borji M, Malekian A, Salajegheh A, Ghadimi M (2016) Multi-time-scale analysis of hydrological drought forecasting using support vector regression (SVR) and artificial neural networks (ANN). Arab J Geosci 9:. https://doi.org/10.1007/s12517-016-2750-X

Cai X, Wang X, Jain P, Flannigan MD (2019) Evaluation of Gridded Precipitation Data and Interpolation Methods for Forest Fire Danger Rating in Alberta, Canada. J Geophys Res Atmos 124:3-17. https://doi.org/10.1029/2018JD028754

Chen H, Sun J (2015) Changes in drought characteristics over china using the standardized precipitation evapotranspiration index. J Clim 28:5430-5447. https://doi.org/10.1175/JCLI-D-14-00707.1

Chen S, Shin JY, Kim TW (2017) Probabilistic forecasting of drought: a hidden Markov model aggregated with the RCP 8.5 precipitation projection. Stoch 

$1279-6$

514 Choubin B, Malekian A (2017) Combined gamma and M-test-based ANN and ARIMA models for groundwater fluctuation forecasting in semiarid regions. Environ Earth Sci 76:. https://doi.org/10.1007/s12665-017-6870-8

Choubin B, Malekian A, Golshan M (2016) Application of several data-driven https://doi.org/10.20937/ATM.2016.29.02.02

Deo RC, Şahin M (2015) Application of the Artificial Neural Network model for prediction of monthly Standardized Precipitation and Evapotranspiration Index using hydrometeorological parameters and climate indices in eastern Australia. Atmos Res 161-162:65-81. https://doi.org/10.1016/j.atmosres.2015.03.018

Deo RC, Şahin M (2016) An extreme learning machine model for the simulation of monthly mean streamflow water level in eastern Queensland. Environ Monit Assess 188:1-24. https://doi.org/10.1007/s10661-016-5094-9

Fung KF, Huang YF, Koo CH (2019a) Coupling fuzzy-SVR and boosting-SVR models with wavelet decomposition for meteorological drought prediction. Environ Earth Sci 78:1-18. https://doi.org/10.1007/s12665-019-8700-7

Fung KF, Huang YF, Koo CH, Soh YW (2019b) Drought forecasting: A review of modelling approaches 2007-2017. J Water Clim Chang. https://doi.org/10.2166/wcc.2019.236 
533 Gao Y, Markkanen T, Thum T, et al (2016) Assessing various drought indicators in 534 representing summer drought in boreal forests in Finland. Hydrol Earth Syst Sci 20:175-191. https://doi.org/10.5194/hess-20-175-2016

536 Gu Y, Brown JF, Verdin JP, Wardlow B (2007) A five-year analysis of MODIS NDVI and NDWI for grassland drought assessment over the central Great Plains of the United States. Geophys Res Lett 34:1-6. https://doi.org/10.1029/2006GL029127

540 Han P, Wang PX, Zhang SY, Zhu DH (2010) Drought forecasting based on the https://doi.org/10.1016/j.mcm.2009.10.031

543 Hayes MJ, Wilhite DA, Vanyarkho O V (1996) Monitoring the 1996 Drought Using 544 the Standardized Precipitation Index. Bull Am Meteorol Soc 429-438

545 Hochreiter S (1997) Long Short-Term Memory. 1780:1735-1780

$546 \mathrm{Hu}$ W, Tong S, Mengersen K, Connell D (2007) Weather Variability and the 547 Incidence of Cryptosporidiosis: Comparison of Time Series Poisson Regression $548 \quad$ and SARIMA Models. Ann Epidemiol 17:679-688.

$549 \quad$ https://doi.org/10.1016/j.annepidem.2007.03.020

550 Jain P, Flannigan MD (2017) Comparison of methods for spatial interpolation of fire 551 weather in Alberta, Canada. Can J For Res 47:1646-1658.

$552 \quad$ https://doi.org/10.1139/cjfr-2017-0101 
553 Jeong J, Park E (2019) Comparative applications of data-driven models representing

$554 \quad$ water table fluctuations. J Hydrol 572:261-273.

$555 \quad$ https://doi.org/10.1016/j.jhydrol.2019.02.051

556 Karavitis CA, Chortaria C, Alexandris S, et al (2012) Development of the

557 standardised precipitation index for Greece. Urban Water J 9:401-417.

$558 \quad$ https://doi.org/10.1080/1573062X.2012.690431

559 Karthika M, Krishnaveni, Thirunavukkarasu V (2017) Forecasting of meteorological

560 drought using ARIMA model. Indian J Agric Res 51:103-111.

$561 \quad$ https://doi.org/10.18805/ijare.v0iOF.7631

562 Kisi O, Sanikhani H, Zounemat-Kermani M, Niazi F (2015) Long-term monthly

563 evapotranspiration modeling by several data-driven methods without climatic

564 data. Comput Electron Agric 115:66-77.

$565 \quad$ https://doi.org/10.1016/j.compag.2015.04.015

566 Kousari MR, Hosseini ME, Ahani H, Hakimelahi H (2017) Introducing an operational

567 method to forecast long-term regional drought based on the application of

$568 \quad$ artificial intelligence capabilities. Theor Appl Climatol 127:361-380.

$569 \quad$ https://doi.org/10.1007/s00704-015-1624-6

570 Li L, She D, Zheng H, et al (2020) Elucidating Diverse Drought Characteristics from

571 Two Meteorological Drought Indices (SPI and SPEI) in China. J Hydrometeorol

21:1513-1530. https://doi.org/10.1175/jhm-d-19-0290.1 
573

574

575

576

577

578

579

580

581

582

583

584

585

586

587

588

589

590

591

592

593

Manatsa D, Chingombe W, Matarira CH (2008) The impact of the positive Indian Ocean dipole on Zimbabwe droughts Tropical climate is understood to be dominated by. Int J Climatol 2029:2011-2029. https://doi.org/10.1002/joc

Marj AF, Meijerink AMJ (2011) Agricultural drought forecasting using satellite images, climate indices and artificial neural network. Int J Remote Sens 32:9707-9719. https://doi.org/10.1080/01431161.2011.575896

Mckee TB, Doesken NJ, Kleist J (1993) The relationship of drought frequency and duration to time scales. Eighth Conf. Appl. Climatol. 17-22 January 1993, Anaheim, Calif. 179:17-22

Mishra AK, Desai VR (2005) Drought forecasting using stochastic models. Stoch Environ Res Risk Assess 19:326-339. https://doi.org/10.1007/s00477-005-02384

Mishra AK, Desai VR (2006) Drought forecasting using feed-forward recursive neural network. Ecol Modell 198:127-138. https://doi.org/10.1016/j.ecolmodel.2006.04.017

Mishra AK, Desai VR, Singh VP (2007) Drought Forecasting Using a Hybrid Stochastic and Neural Network Model. J Hydrol Eng 12:626-638. https://doi.org/10.1061/(ASCE)1084-0699(2007)12:6(626)

Modaresi F, Araghinejad S, Ebrahimi K (2018) A Comparative Assessment of Artificial Neural Network, Generalized Regression Neural Network, LeastSquare Support Vector Regression, and K-Nearest Neighbor Regression for 
Mossad A, Alazba AA (2015) Drought forecasting using stochastic models in a hyperarid climate. Atmosphere (Basel) 6:410-430. https://doi.org/10.3390/atmos6040410

Narasimhan B, Srinivasan R (2005) Development and evaluation of Soil Moisture Deficit Index (SMDI) and Evapotranspiration Deficit Index (ETDI) for agricultural drought monitoring. Agric For Meteorol 133:69-88.

602 https://doi.org/10.1016/j.agrformet.2005.07.012

Ochoa-Rivera JC (2008) Prospecting droughts with stochastic artificial neural networks. J Hydrol 352:174-180. https://doi.org/10.1016/j.jhydrol.2008.01.006

Paulo AA, Rosa RD, Pereira LS (2012) Climate trends and behaviour of drought indices based on precipitation and evapotranspiration in Portugal. Nat Hazards Earth Syst Sci 12:1481-1491. https://doi.org/10.5194/nhess-12-1481-2012

Poornima S, Pushpalatha M (2019) Drought prediction based on SPI and SPEI with varying timescales using LSTM recurrent neural network. Soft Comput 1:.

611 Qi Y, Li Q, Karimian H, Liu D (2019) A hybrid model for spatiotemporal forecasting 612 of PM 2.5 based on graph convolutional neural network and long short-term 613 memory. Sci Total Environ 664:1-10.

614 https://doi.org/10.1016/j.scitotenv.2019.01.333 
615 Rafiei-Sardooi E, Mohseni-Saravi M, Barkhori S, et al (2018) Drought modeling: a

616 comparative study between time series and neuro-fuzzy approaches. Arab J

617 Geosci 11:. https://doi.org/10.1007/s12517-018-3835-5

618 Salman AG, Heryadi Y, Abdurahman E, Suparta W (2018) Weather forecasting using

619 merged Long Short-Term Memory Model (LSTM) and Autoregressive

620 Integrated Moving Average (ARIMA) Model. J Comput Sci 14:930-938.

$621 \quad$ https://doi.org/10.3844/jcssp.2018.930.938

622 Seibert M, Merz B, Apel H (2017) Seasonal forecasting of hydrological drought in the

623 Limpopo Basin: A comparison of statistical methods. Hydrol Earth Syst Sci

624

21:1611-1629. https://doi.org/10.5194/hess-21-1611-2017

625 Sigaroodi SK, Chen Q, Ebrahimi S, et al (2013) Long-term precipitation forecast for

626 drought relief using atmospheric circulation factors: a study on the Maharloo

627 Basin in Iran. Hydrol Earth Syst Sci Discuss 10:13333-13361.

628 https://doi.org/10.5194/hessd-10-13333-2013

629 Soh YW, Koo CH, Huang YF, Fung KF (2018) Application of artificial intelligence

630 models for the prediction of standardized precipitation evapotranspiration index

631 (SPEI) at Langat River Basin, Malaysia. Comput Electron Agric 144:164-173.

632 https://doi.org/10.1016/j.compag.2017.12.002

633 Suykens JAK, De Brabanter J, Lukas L, Vandewalle J (2002) Weighted least squares

634 support vector machines: Robustness and sparce approximation.

635 Neurocomputing 48:85-105. https://doi.org/10.1016/S0925-2312(01)00644-0 
636 Tatli H, Türkeş M (2011) Empirical Orthogonal Function analysis of the palmer

637 drought indices. Agric For Meteorol 151:981-991.

638 https://doi.org/10.1016/j.agrformet.2011.03.004

639 Tian M, Wang P, Khan J (2016) Drought forecasting with vegetation temperature

640 condition index using arima models in the guanzhong plain. Remote Sens 8:.

$641 \quad$ https://doi.org/10.3390/rs8090690

642 Tian Y, Xu YP, Wang G (2018) Agricultural drought prediction using climate indices

643 based on Support Vector Regression in Xiangjiang River basin. Sci Total

644 Environ 622-623:710-720. https://doi.org/10.1016/j.scitotenv.2017.12.025

645 Tran Anh D, Van SP, Dang TD, Hoang LP (2019) Downscaling rainfall using deep

646 learning long short-term memory and feedforward neural network. Int J

647 Climatol. https://doi.org/10.1002/joc.6066

648 Vicente-Serrano SM, Beguería S, López-Moreno JI (2010) A multiscalar drought

649 index sensitive to global warming: The standardized precipitation

650 evapotranspiration index. J Clim 23:1696-1718.

$651 \quad$ https://doi.org/10.1175/2009JCLI2909.1

652 Vicente-Serrano SM, Van der Schrier G, Beguería S, et al (2015) Contribution of

653 precipitation and reference evapotranspiration to drought indices under different

654 climates. J Hydrol 526:42-54. https://doi.org/10.1016/j.jhydrol.2014.11.025

655 Wang H, Vicente-serrano SM, Tao F, et al (2016) Monitoring winter wheat drought

656 threat in Northern China using multiple climate-based drought indices and soil 
Wang Q, Shi P, Lei T, et al (2015) The alleviating trend of drought in the HuangHuai-Hai Plain of China based on the daily SPEI. Int J Climatol 35:3760-3769.

661 https://doi.org/10.1002/joc.4244

662

Xu D, Zhang Q, Ding Y, Huang H (2020a) Application of a hybrid ARIMA-SVR model based on the SPI for the forecast of drought—A case study in Henan Province, China. J Appl Meteorol Climatol. https://doi.org/10.1175/jamc-d-19-

665 0270.1

666

Xu D, Zhang Q, Ding Y, Huang H (2020b) Application of a Hybrid ARIMA-SVR Model Based on the SPI for the Forecast of Drought - A Case Study in Henan Province, China. J Appl Meteorol Climatol 59:1239-1259.

669 https://doi.org/10.1175/jamc-d-19-0270.1

670 Yao J, Zhao Y, Chen Y, et al (2018a) Multi-scale assessments of droughts: A case 671 study in Xinjiang, China. Sci Total Environ 630:444-452.

672 https://doi.org/10.1016/j.scitotenv.2018.02.200

673 Yao N, Li Y, Lei T, Peng L (2018b) Drought evolution, severity and trends in 674 mainland China over 1961-2013. Sci Total Environ 616-617:73-89.

675 https://doi.org/10.1016/j.scitotenv.2017.10.327 
676

678

686

Yurekli K, Kurunc A, Ozturk F (2005) Application of linear stochastic models to monthly flow data of Kelkit Stream. Ecol Modell 183:67-75. https://doi.org/10.1016/j.ecolmodel.2004.08.001

Zhang J, Zhu Y, Zhang X, et al (2018) Developing a Long Short-Term Memory (LSTM) based model for predicting water table depth in agricultural areas. J Hydrol 561:918-929. https://doi.org/10.1016/j.jhydrol.2018.04.065

Zhang R, Chen ZY, Xu LJ, Ou CQ (2019) Meteorological drought forecasting based on a statistical model with machine learning techniques in Shaanxi province, China. Sci Total Environ 665:338-346. https://doi.org/10.1016/j.scitotenv.2019.01.431

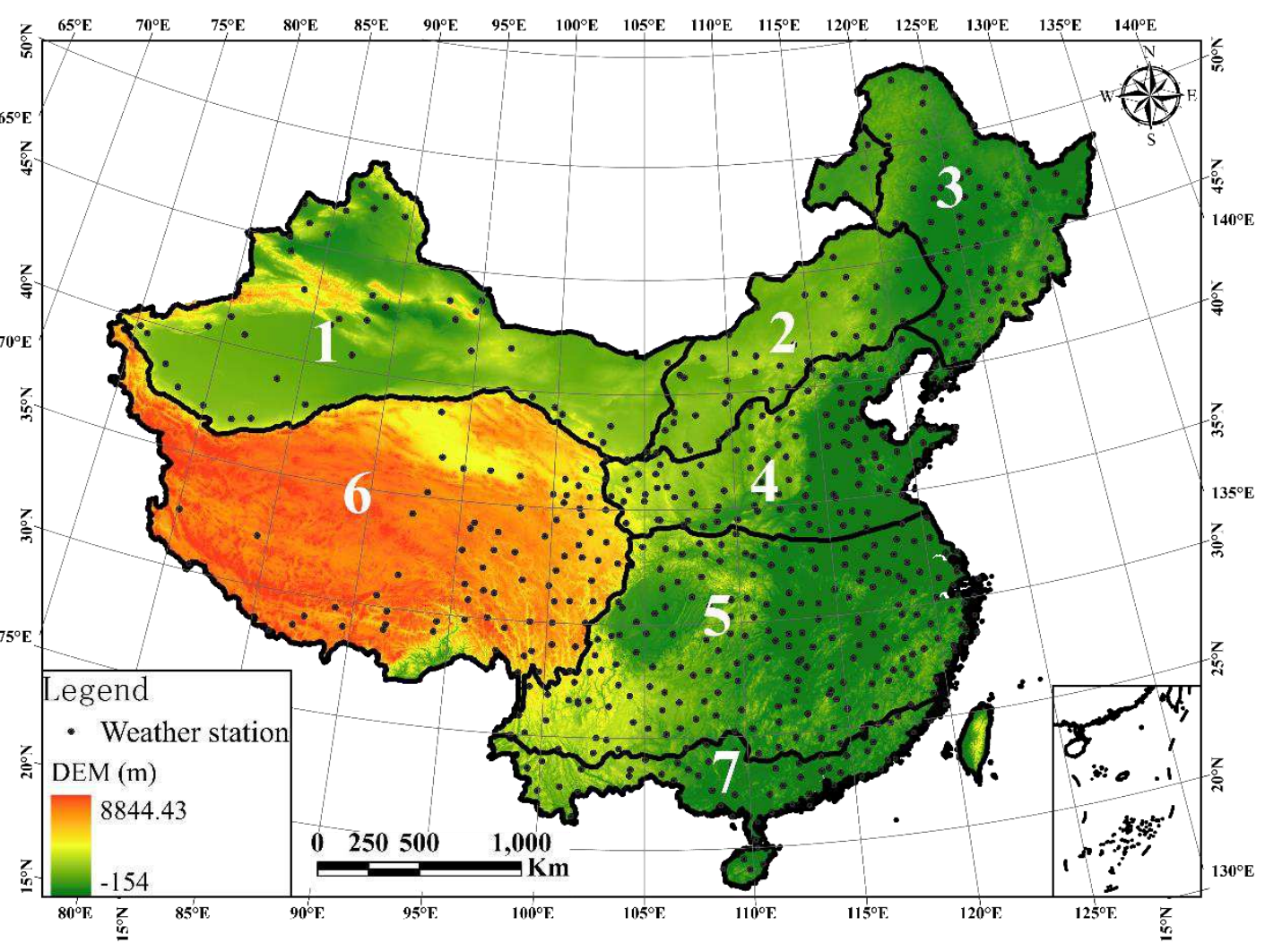


689 China. The numbers in the map denotes different sub-regions.

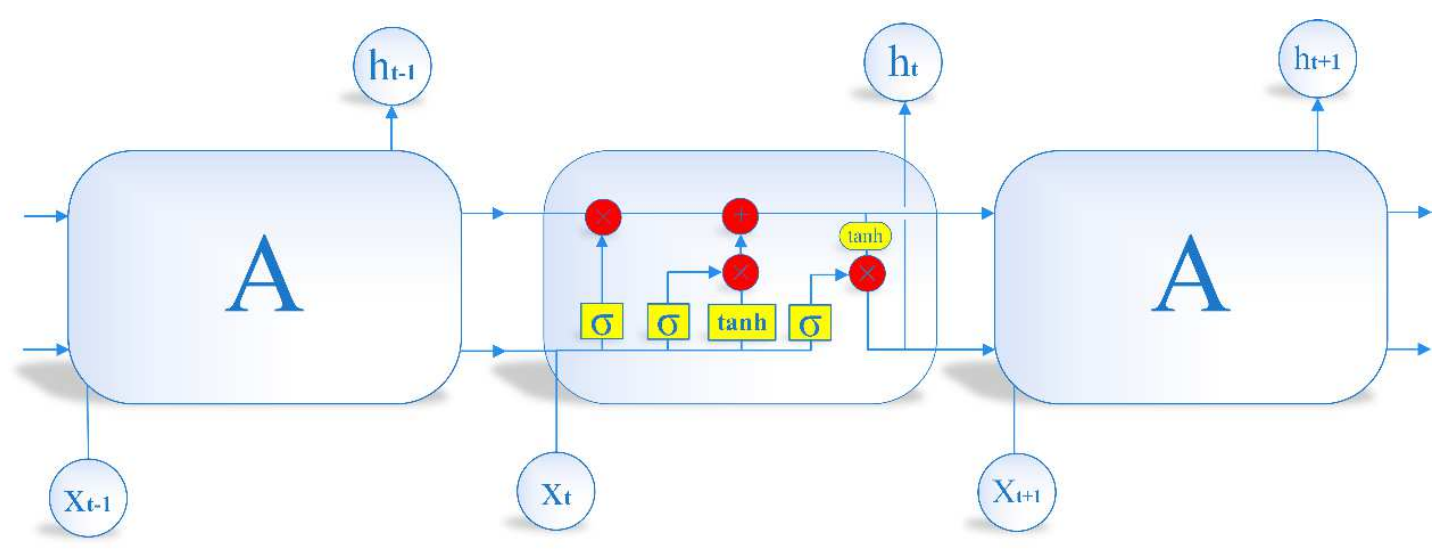

691 Fig.2. Four interaction layers of the LSTM module chain.

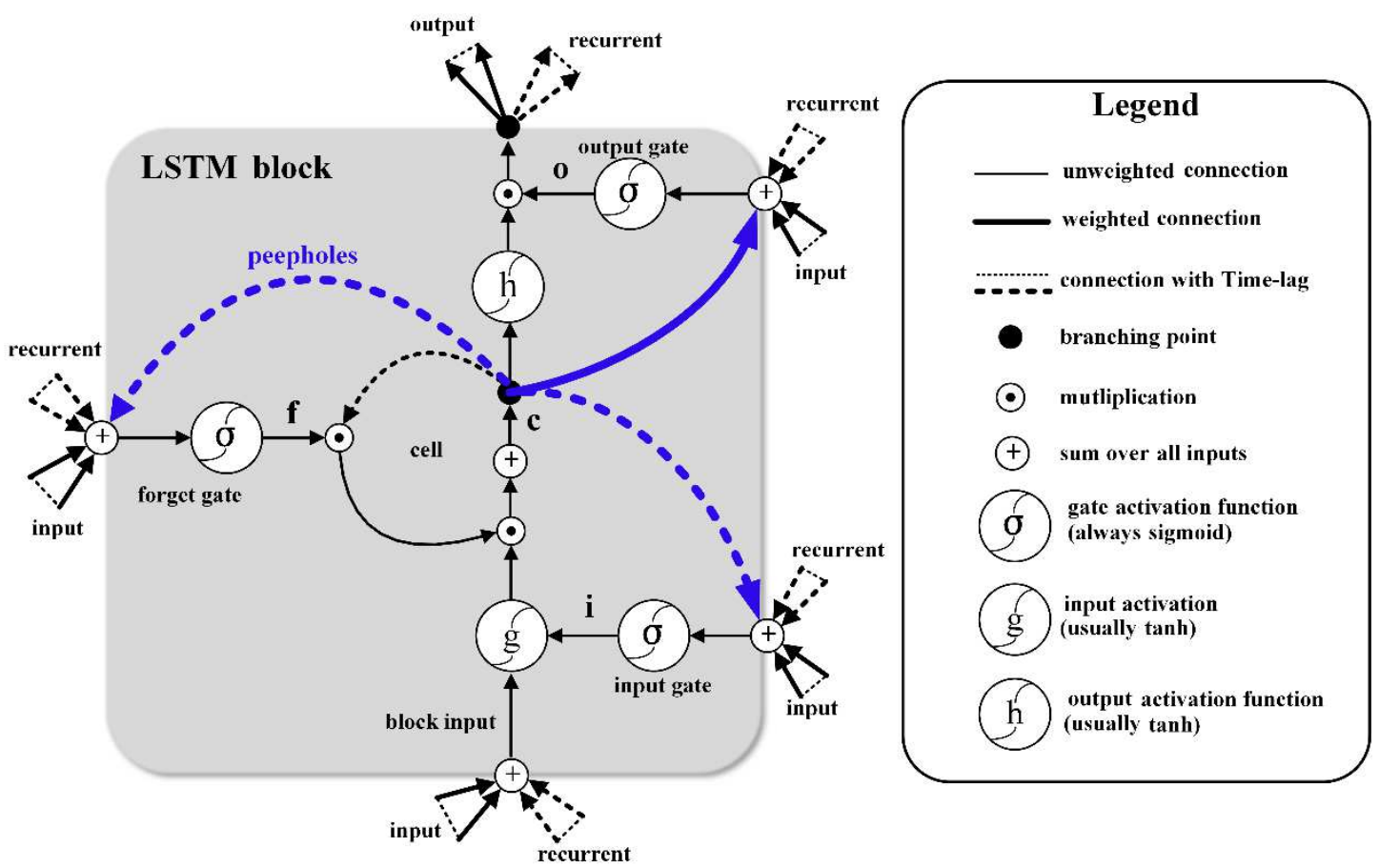

Fig.3. LSTM cell structure in hidden layer. 


\section{Hybrid ARIMA-LSTM Model}

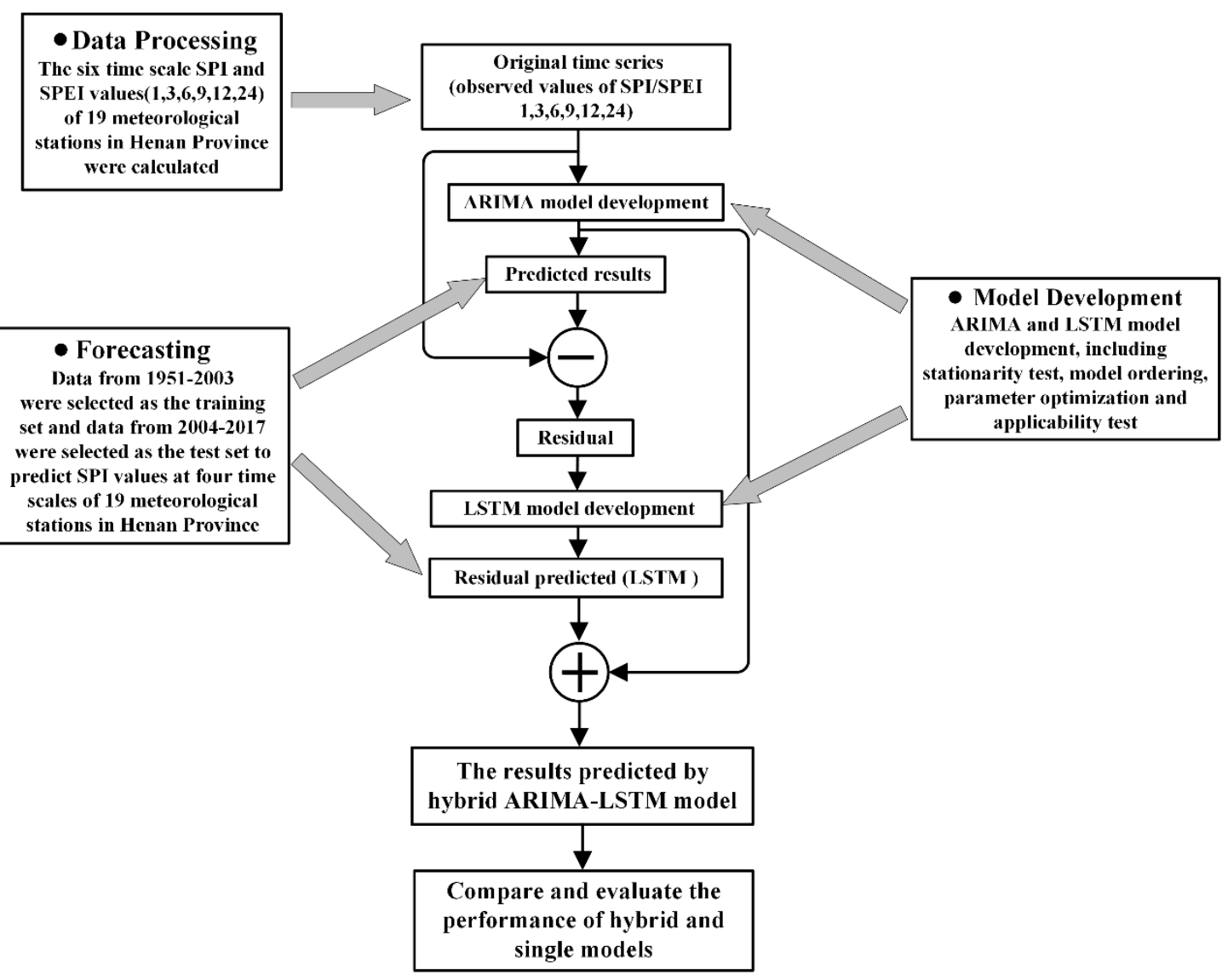

696 Fig.4. hybrid ARIMA-LSTM model flow chart.

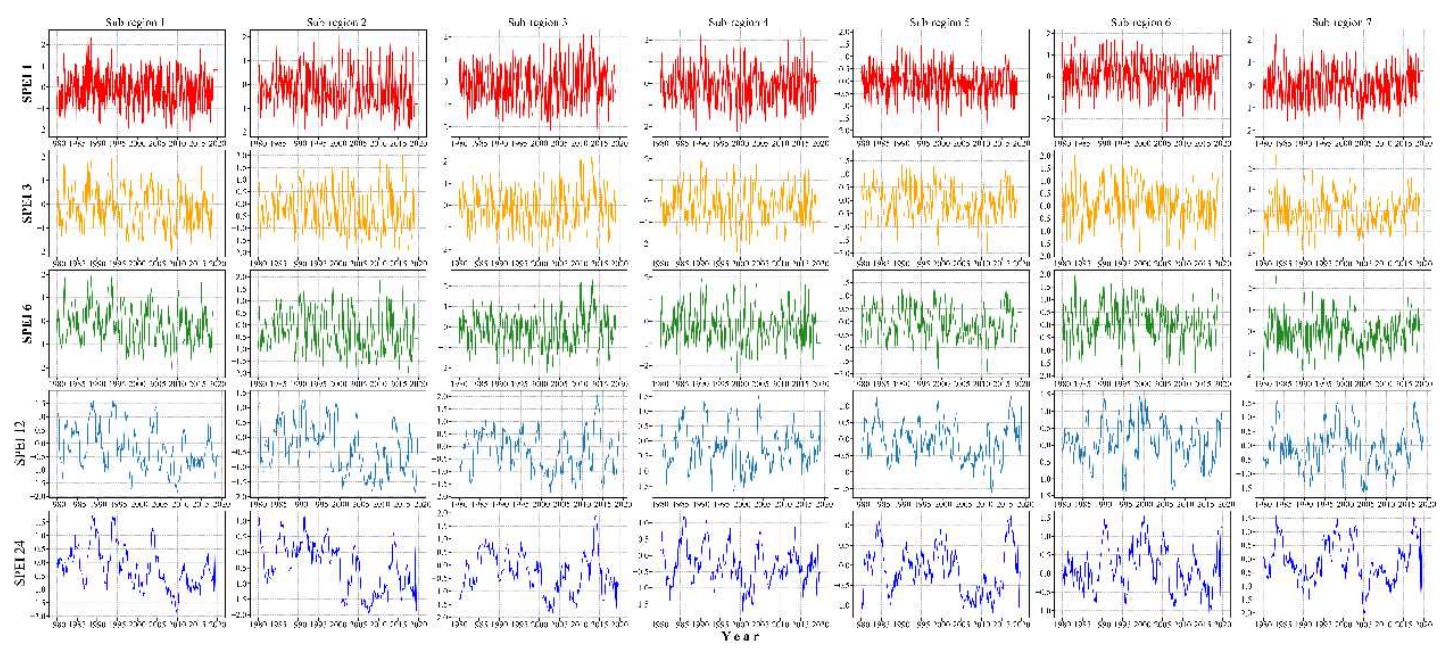

698 Fig.5. The variations of SPEI at the 1-, 3-, 6-, 12- and 24-month timescales over 

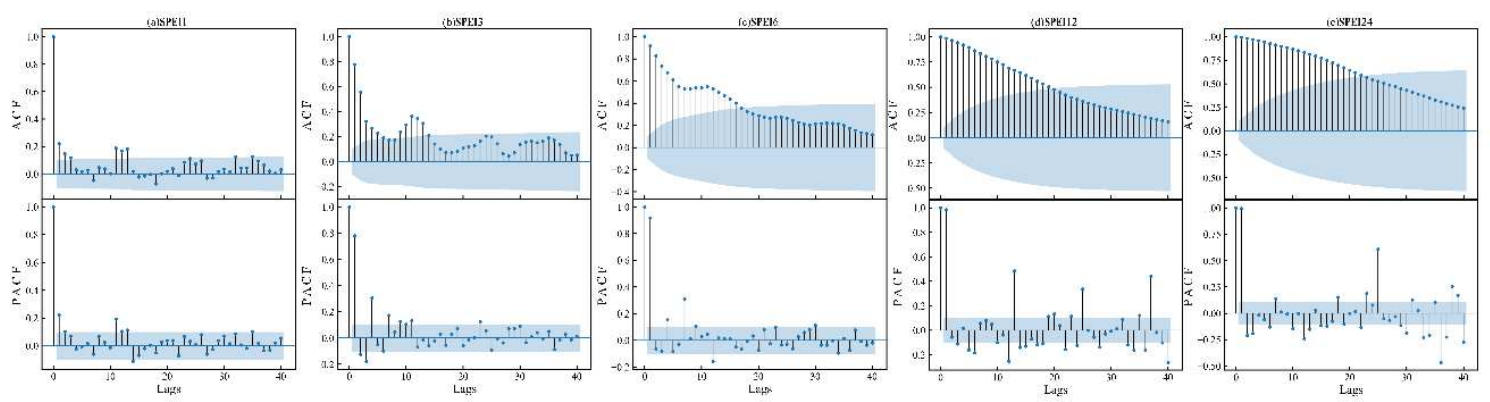

702

703 Fig.6. ACF and PACF of SPEI at the 1-, 3-, 6-, 12- and 24-month timescales in sub-

704 region 1 of China.

705
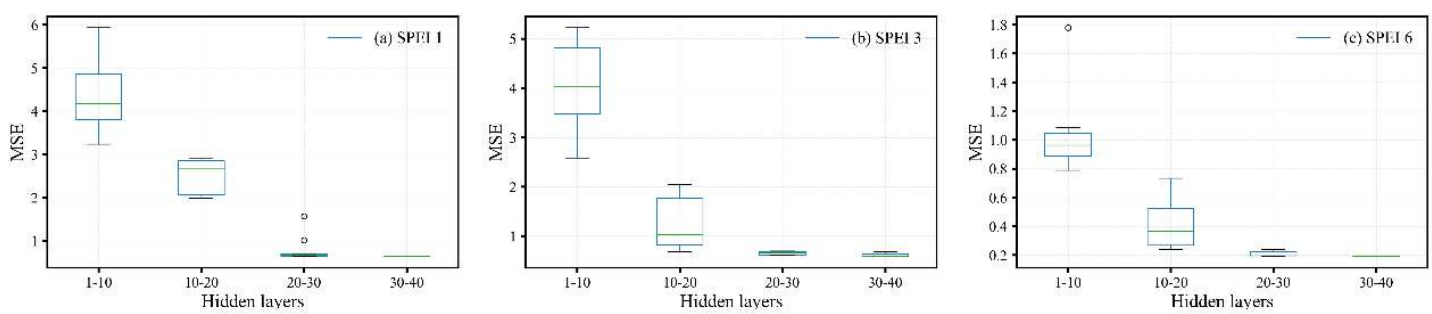

706
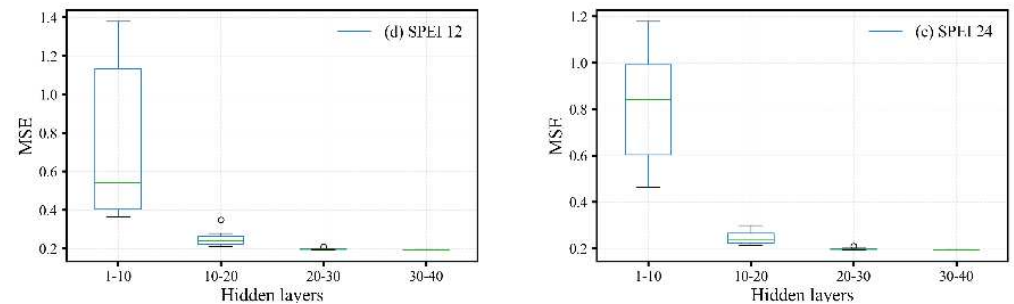

707 Fig.7 Multitime scale boxplot of loss fuction and hidden layers of SPEI in sub-region 7081 of China.

709 

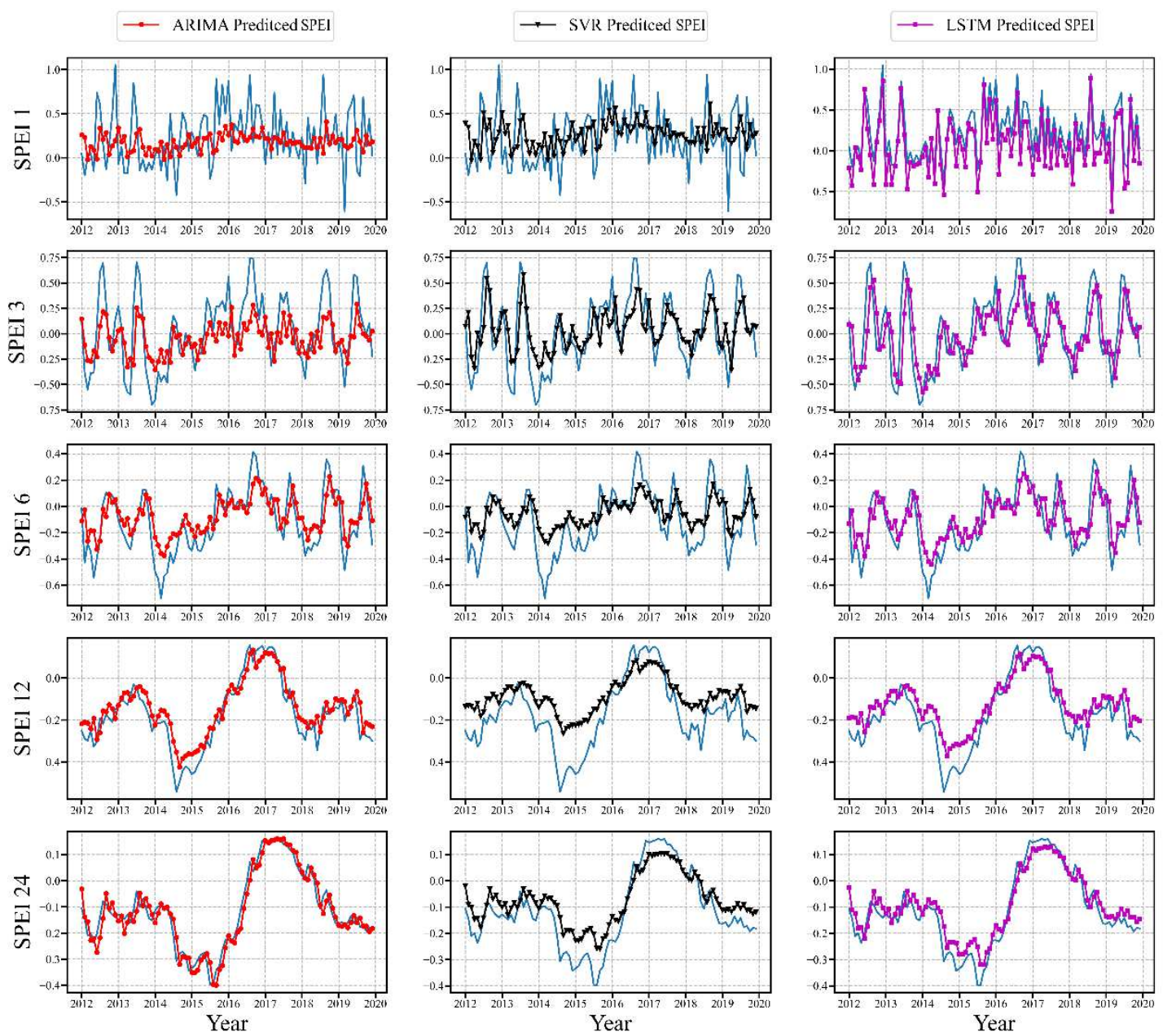

712 Fig.8. Forecast of multitime scale SPEI value of ARIMA model, SVR model and

713 LSTM model. 

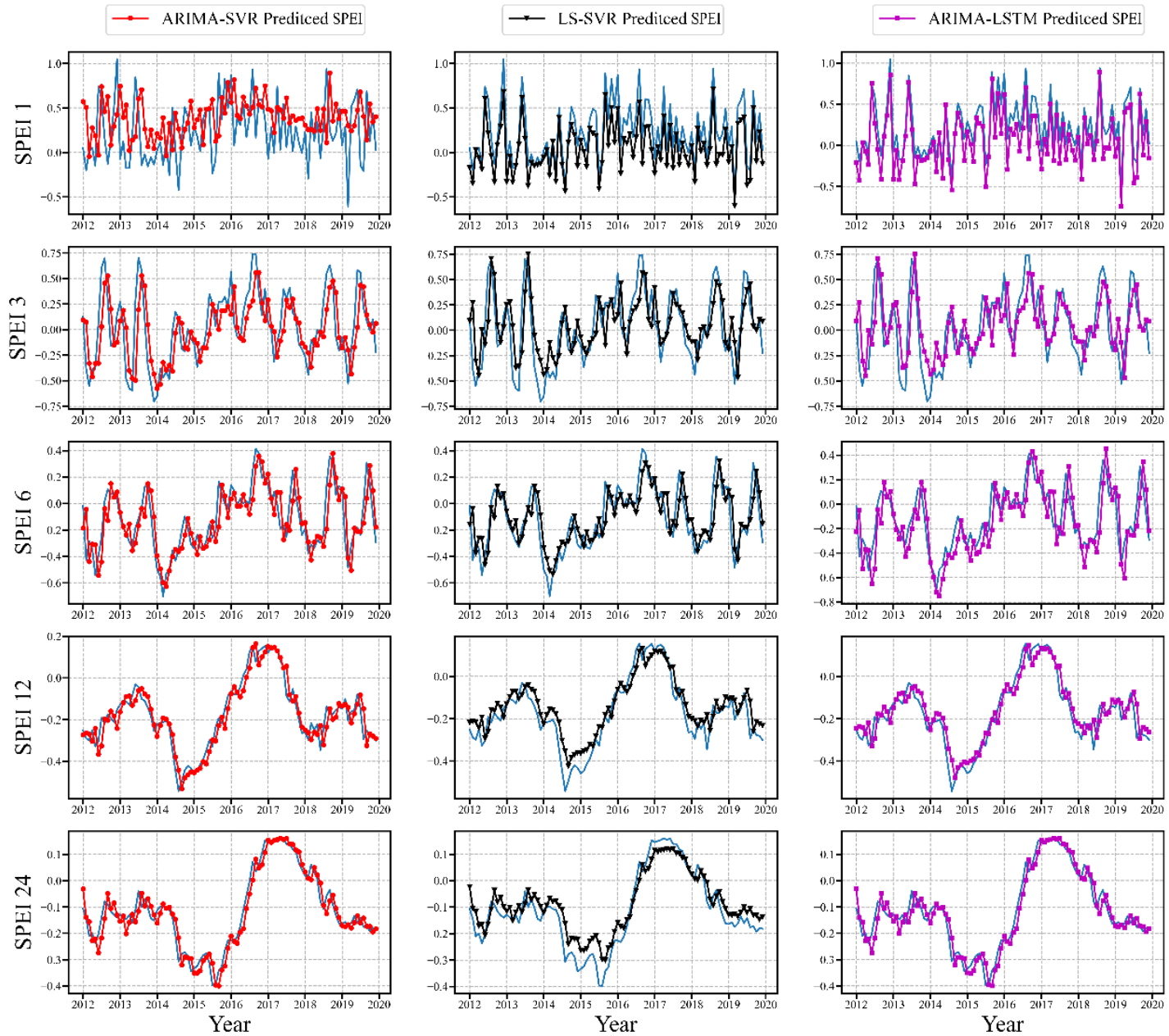

Fig.9. Forecast of multitime scale SPEI value of hybrid ARIMA-SVR model, LS-

721 SVR model and ARIMA-LSTM model. 


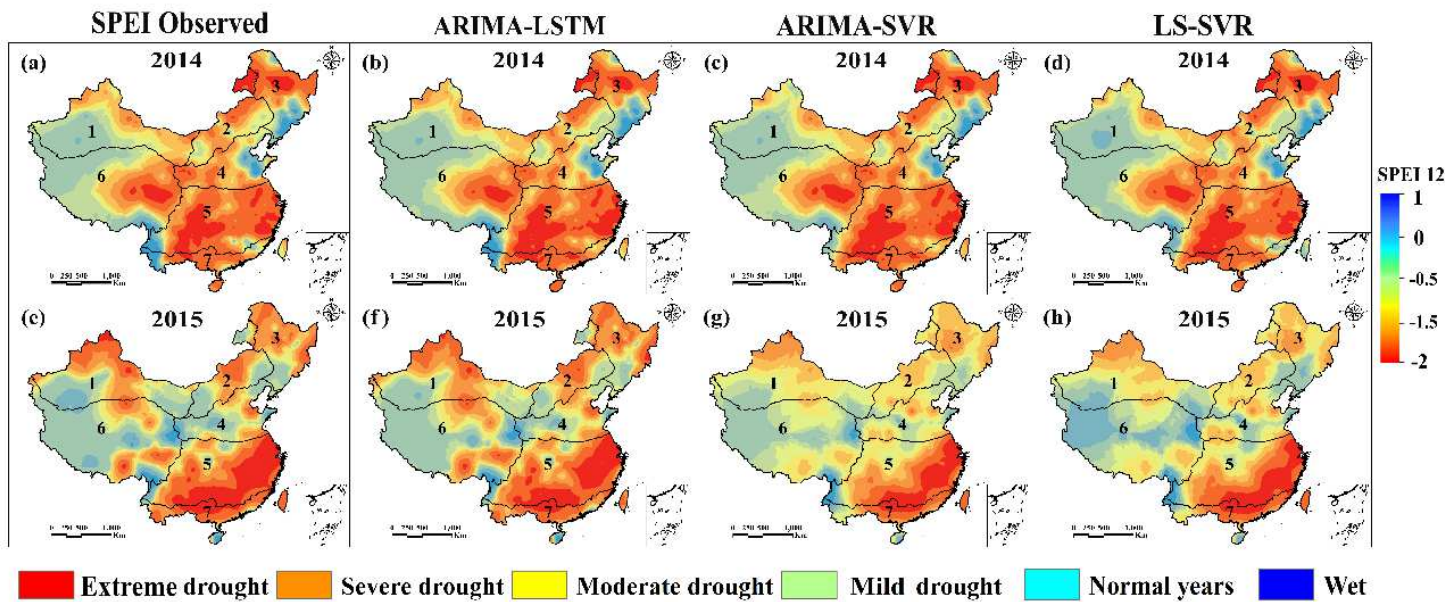

724 Fig.10. Spatial distributions of SPEI drought levels in 2014 and 2015, using the three

725 forecasting models (ARIMA-SVR, LS-SVR and ARIMA-LSTM model).

\section{Table 1}

728 Drought level classification using SPEI.

\begin{tabular}{lll}
\hline Level & Type & Range of SPEI \\
\hline 0 & Wet & $0.5 \leq$ SPEI \\
1 & No drought & $-0.5 \leq$ SPEI $<0.5$ \\
2 & Mild drought & $-1.0 \leq$ SPEI $<-0.5$ \\
3 & Moderate drought & $-1.5 \leq$ SPEI $<-1.0$ \\
4 & Severe drought & $-2.0 \leq$ SPEI $<-1.5$ \\
5 & Extreme drought & SPEI $\leq-2.0$ \\
\hline
\end{tabular}


730 Table 2

731 Unit root test of 7 sub-regions at five time scales of the SPEI original sequence.

\begin{tabular}{|c|c|c|c|c|c|c|c|c|c|c|c|c|c|c|}
\hline \multirow{2}{*}{$\begin{array}{c}\text { SPEI } \\
\text { series }\end{array}$} & \multicolumn{2}{|c|}{ Sub-region 1} & \multicolumn{2}{|c|}{ Sub-region2 } & \multicolumn{2}{|c|}{ Sub-region3 } & \multicolumn{2}{|c|}{ Sub-region 4} & \multicolumn{2}{|c|}{ Sub-region5 } & \multicolumn{2}{|c|}{ Sub-region6 } & \multicolumn{2}{|c|}{ Sub-region7 } \\
\hline & $\mathrm{ADF}$ & P-value & $\mathrm{ADF}$ & P-value & $\mathrm{ADF}$ & P-value & $\mathrm{ADF}$ & P-value & $\mathrm{ADF}$ & P-value & $\mathrm{ADF}$ & P-value & $\mathrm{ADF}$ & P-value \\
\hline SPEI-1 & -3.834 & $2.57 \mathrm{E}-03$ & -17.395 & 4.97E-30 & -12.979 & $2.97 \mathrm{E}-24$ & -17.759 & $3.34 \mathrm{E}-30$ & -18.672 & 2.04E-30 & -18.006 & $2.72 \mathrm{E}-30$ & -17.46 & $4.58 \mathrm{E}-30$ \\
\hline SPEI-3 & -2.803 & $5.78 \mathrm{E}-02$ & -9.068 & $4.36 \mathrm{E}-15$ & -7.654 & $1.75 \mathrm{E}-11$ & -8.092 & $1.36 \mathrm{E}-12$ & -7.513 & 3.94E-11 & -4.337 & $3.81 \mathrm{E}-04$ & -6.842 & $1.78 \mathrm{E}-09$ \\
\hline SPEI-6 & -2.587 & $9.55 \mathrm{E}-02$ & -4.777 & 6.01E-05 & -6.398 & $2.02 \mathrm{E}-08$ & -4.217 & $6.10 \mathrm{E}-04$ & -4.545 & $1.62 \mathrm{E}-04$ & -5.281 & $5.97 \mathrm{E}-06$ & -3.765 & $3.27 \mathrm{E}-03$ \\
\hline SPEI-12 & -2.671 & $7.90 \mathrm{E}-02$ & -2.342 & $1.59 \mathrm{E}-01$ & -3.5 & $7.98 \mathrm{E}-03$ & -2.656 & $8.18 \mathrm{E}-02$ & -3.664 & 4.64E-03 & -6.953 & $9.56 \mathrm{E}-10$ & -3.584 & $6.07 \mathrm{E}-03$ \\
\hline SPEI-24 & -2.335 & $1.61 \mathrm{E}-01$ & -2.026 & $2.75 \mathrm{E}-01$ & -3.738 & $3.60 \mathrm{E}-03$ & -2.53 & 0.10829 & -4.694 & $8.62 \mathrm{E}-05$ & -4.462 & $2.29 \mathrm{E}-04$ & -3.014 & $3.36 \mathrm{E}-02$ \\
\hline
\end{tabular}

732 
Table 3

AIC, BIC,HQIC and PLjung-Box test comparisons of selected models at five-time scale of

736 SPEI for 7 sub-regions.

\begin{tabular}{|c|c|c|c|c|c|c|}
\hline $\begin{array}{l}\text { SPEI } \\
\text { series }\end{array}$ & Example Stations & Model & AIC & BIC & HQIC & $\mathrm{P}_{\text {Ljung-Box }}$ \\
\hline \multirow[t]{7}{*}{ SPEI-1 } & Sub-region1 & $\operatorname{ARIMA}(3,0,5)(1,0,2)_{1}$ & 2248.092 & 234.352 & 2269.701 & 0.996 \\
\hline & Sub-region2 & $\operatorname{ARIMA}(3,0,3)(0,0,1)_{1}$ & 719.718 & 753.092 & 732.838 & 0.886 \\
\hline & Sub-region3 & $\operatorname{ARIMA}(3,0,5)(1,0,0)_{1}$ & 905.207 & 917.722 & 910.127 & 0.981 \\
\hline & Sub-region4 & $\operatorname{ARIMA}(3,0,3)(1,0,0)_{1}$ & 732.199 & 744.714 & 737.119 & 0.985 \\
\hline & Sub-region5 & $\operatorname{ARIMA}(4,0,2)(0,0,1)_{1}$ & 594.122 & 627.495 & 607.241 & 0.945 \\
\hline & Sub-region6 & $\operatorname{ARIMA}(3,0,5)(1,0,0)_{1}$ & 293.753 & 306.286 & 298.673 & 0.914 \\
\hline & Sub-region7 & $\operatorname{ARIMA}(1,0,0)_{1}$ & 829.772 & 842.287 & 834.692 & 0.959 \\
\hline \multirow[t]{7}{*}{ SPEI-3 } & Sub-region1 & $\operatorname{ARIMA}(2,0,3)(1,0,3)_{3}$ & 156.527 & 181.556 & 166.367 & 0.457 \\
\hline & Sub-region2 & $\operatorname{ARIMA}(5,0,4)(0,0,2)_{3}$ & 335.71 & 381.599 & 353.749 & 0.994 \\
\hline & Sub-region3 & $\operatorname{ARIMA}(2,0,4)(0,0,2)_{3}$ & 565.241 & 581.928 & 571.801 & 0.716 \\
\hline & Sub-region4 & $\operatorname{ARIMA}(1,0,2)_{3}$ & 335.785 & 356.644 & 343.985 & 0.81 \\
\hline & Sub-region5 & $\operatorname{ARIMA}(5,0,4)(2,0,0)_{3}$ & 255.756 & 272.443 & 262.316 & 0.315 \\
\hline & Sub-region6 & $\operatorname{ARIMA}(2,0,5)(1,0,3)_{3}$ & 94.575 & 119.605 & 104.415 & 0.227 \\
\hline & Sub-region7 & $\operatorname{ARIMA}(0,0,2)(0,0,3)_{3}$ & 389.353 & 410.211 & 397.552 & 0.939 \\
\hline \multirow[t]{7}{*}{ SPEI-6 } & Sub-region 1 & $\operatorname{ARIMA}(8,0,2)(8,0,1)_{6}$ & 116.904 & 66.844 & 97.225 & 0.77 \\
\hline & Sub-region2 & $\operatorname{ARIMA}(9,0,5)(1,0,5)_{6}$ & 11.284 & 22.126 & 1.872 & 0.947 \\
\hline & Sub-region3 & $\operatorname{ARIMA}(0,0,5)_{6}$ & 232.062 & 261.264 & 243.542 & 0.891 \\
\hline & Sub-region4 & $\operatorname{ARIMA}(2,0,5)(1,0,5)_{6}$ & 52.585 & 19.212 & 39.466 & 0.785 \\
\hline & Sub-region5 & $\operatorname{ARIMA}(0,0,5)_{6}$ & 113.02 & 83.818 & 101.541 & 0.824 \\
\hline & Sub-region6 & $\operatorname{ARIMA}(9,0,5)(7,0,0)_{6}$ & 175.86 & 138.314 & 161.1 & 0.693 \\
\hline & Sub-region7 & $\operatorname{ARIMA}(0,0,5)_{6}$ & 63.574 & 92.776 & 75.054 & 0.541 \\
\hline \multirow[t]{7}{*}{ SPEI-12 } & Sub-region1 & $\operatorname{ARIMA}(10,0,5)(9,0,5)_{12}$ & 473.387 & 419.155 & 452.067 & 0.899 \\
\hline & Sub-region2 & $\operatorname{ARIMA}(10,0,5)_{12}$ & 574.376 & 520.144 & 553.057 & 0.975 \\
\hline & Sub-region3 & $\operatorname{ARIMA}(10,0,5)_{12}$ & 335.364 & 281.132 & 314.044 & 0.935 \\
\hline & Sub-region4 & $\operatorname{ARIMA}(10,0,5)(7,0,5)_{12}$ & 532.11 & 477.878 & 510.791 & 0.969 \\
\hline & Sub-region5 & $\operatorname{ARIMA}(8,0,4)(4,0,2)_{12}$ & 499.37 & 449.309 & 479.69 & 0.666 \\
\hline & Sub-region6 & $\operatorname{ARIMA}(10,0,5)(1,0,0)_{12}$ & 399.511 & 349.45 & 379.831 & 0.85 \\
\hline & Sub-region7 & $\operatorname{ARIMA}(10,0,5)_{12}$ & 259.529 & 209.468 & 239.849 & 0.856 \\
\hline \multirow[t]{7}{*}{ SPEI-24 } & Sub-region1 & $\operatorname{ARIMA}(8,0,5)(2,0,1)_{24}$ & 625.846 & 604.987 & 617.646 & 0.927 \\
\hline & Sub-region2 & $\operatorname{ARIMA}(10,0,4)(1,0,0)_{24}$ & 954.879 & 555.132 & 928.64 & 0.967 \\
\hline & Sub-region3 & $\operatorname{ARIMA}(7,0,4)(1,0,0)_{24}$ & 655.885 & 601.653 & 634.566 & 0.952 \\
\hline & Sub-region4 & $\operatorname{ARIMA}(6,0,4)(1,0,0)_{24}$ & 775.902 & 763.386 & 770.982 & 0.485 \\
\hline & Sub-region5 & $\operatorname{ARIMA}(10,0,4)(3,0,2)_{24}$ & 701.918 & 672.716 & 690.438 & 0.72 \\
\hline & Sub-region6 & $\operatorname{ARIMA}(8,0,5)(1,0,0)_{24}$ & 162.256 & 149.741 & 157.336 & 0.899 \\
\hline & Sub-region7 & $\operatorname{ARIMA}(10,0,4)(2,0,0)_{24}$ & 578.841 & 562.155 & 572.282 & 0.941 \\
\hline
\end{tabular}


Table 4

739 Performance measures for comparison of observed and predicted data for 1-2 month lead 740 times for five-time scale of SPEI.

741

\begin{tabular}{lllrrrrr}
\hline $\begin{array}{l}\text { Lead } \\
\text { times }\end{array}$ & Model & Performance & SPEI1 & SPEI3 & SPEI6 & SPEI-12 & SPEI-24 \\
\hline 1-month & ARIMA & MSE & 0.431 & 0.535 & 0.277 & 0.246 & 0.187 \\
& & NSE & -0.055 & 0.146 & 0.389 & 0.611 & 0.683 \\
& & RMSE & 0.637 & 0.642 & 0.454 & 0.373 & 0.351 \\
& & MAE & 0.634 & 0.596 & 0.507 & 0.318 & 0.304 \\
& SVR & MSE & 0.314 & 0.409 & 0.667 & 0.636 & 0.577 \\
& & NSE & 0.127 & 0.247 & 0.149 & 0.371 & 0.443 \\
& & RMSE & 0.548 & 0.468 & 0.824 & 0.743 & 0.721 \\
& & MAE & 0.278 & 0.308 & 0.787 & 0.598 & 0.584 \\
& LSTM & MSE & 0.372 & 0.472 & 0.472 & 0.441 & 0.382 \\
& & NSE & 0.036 & 0.196 & 0.269 & 0.491 & 0.563 \\
& & RMSE & 0.592 & 0.555 & 0.639 & 0.558 & 0.536 \\
& MAE & 0.456 & 0.452 & 0.647 & 0.458 & 0.444 \\
& ARIMA & MSE & 1.436 & 1.54 & 1.282 & 1.251 & 1.192 \\
& & NSE & -0.425 & -0.224 & 0.019 & 0.241 & 0.313 \\
& & RMSE & 1.027 & 1.032 & 0.844 & 0.763 & 0.741 \\
& & MAE & 1.024 & 0.986 & 0.897 & 0.708 & 0.694 \\
& SVR & MSE & 0.704 & 0.799 & 1.057 & 1.026 & 0.967 \\
& & NSE & -0.444 & -0.283 & -0.211 & 0.011 & 0.083 \\
& RMSE & 1.052 & 1.015 & 1.099 & 1.018 & 0.996 \\
& & MAE & 0.916 & 0.912 & 1.107 & 0.918 & 0.904 \\
& LSTM & MSE & 1.896 & 1.802 & 1.742 & 1.711 & 1.652 \\
& NSE & -0.414 & -0.253 & -0.181 & 0.041 & 0.113 \\
& & RMSE & 0.902 & 0.865 & 0.949 & 0.868 & 0.846 \\
& & MAE & 0.866 & 0.862 & 1.057 & 0.868 & 0.854 \\
\hline
\end{tabular}

743

744

745

746

747

748

749 
Table 5

753 Performance measures for comparison of observed and predicted data for 1-2 month lead 754 times for five-time scale of SPEI.

\begin{tabular}{lllrrrrr}
\hline $\begin{array}{l}\text { Lead } \\
\text { times }\end{array}$ & \multirow{2}{*}{ Model } & Performance & SPEI1 & SPEI3 & SPEI6 & SPEI-12 & SPEI-24 \\
\hline 1-month & ARIMA-SVR & MSE & 0.282 & 0.386 & 0.128 & 0.097 & 0.038 \\
& & NSE & 0.043 & 0.244 & 0.487 & 0.709 & 0.781 \\
& & RMSE & 0.53 & 0.535 & 0.347 & 0.266 & 0.244 \\
& & MAE & 0.424 & 0.386 & 0.297 & 0.108 & 0.094 \\
& LS-SVR & MSE & 0.264 & 0.276 & 0.249 & 0.148 & 0.107 \\
& & NSE & 0.168 & 0.379 & 0.378 & 0.649 & 0.543 \\
& & RMSE & 0.437 & 0.508 & 0.448 & 0.398 & 0.313 \\
& & MAE & 0.308 & 0.324 & 0.387 & 0.287 & 0.187 \\
& ARIMA-LSTM & MSE & 0.175 & 0.204 & 0.019 & 0.003 & 0.001 \\
& & NSE & 0.368 & 0.451 & 0.659 & 0.885 & 0.93 \\
& & RMSE & 0.347 & 0.479 & 0.137 & 0.053 & 0.037 \\
& MAE & 0.207 & 0.189 & 0.108 & 0.042 & 0.028 \\
& ARIMA-SVR & MSE & 0.772 & 0.876 & 0.618 & 0.587 & 0.528 \\
& & NSE & -0.337 & -0.136 & 0.107 & 0.329 & 0.401 \\
& & RMSE & 1.05 & 1.055 & 0.867 & 0.786 & 0.764 \\
& & MAE & 0.944 & 0.906 & 0.817 & 0.628 & 0.614 \\
& LS-SVR & MSE & 0.784 & 0.796 & 0.769 & 0.668 & 0.627 \\
& & NSE & -0.372 & -0.161 & -0.162 & 0.109 & 0.003 \\
& & RMSE & 0.907 & 0.978 & 0.918 & 0.868 & 0.783 \\
& & MAE & 0.778 & 0.794 & 0.857 & 0.757 & 0.657 \\
& ARIMA-LSTM & MSE & 0.645 & 0.674 & 0.489 & 0.473 & 0.471 \\
& & NSE & 0.068 & 0.151 & 0.359 & 0.585 & 0.63 \\
& & RMSE & 0.817 & 0.949 & 0.607 & 0.523 & 0.507 \\
& & MAE & 0.677 & 0.659 & 0.578 & 0.512 & 0.498 \\
\hline & & & & & &
\end{tabular}


Figures

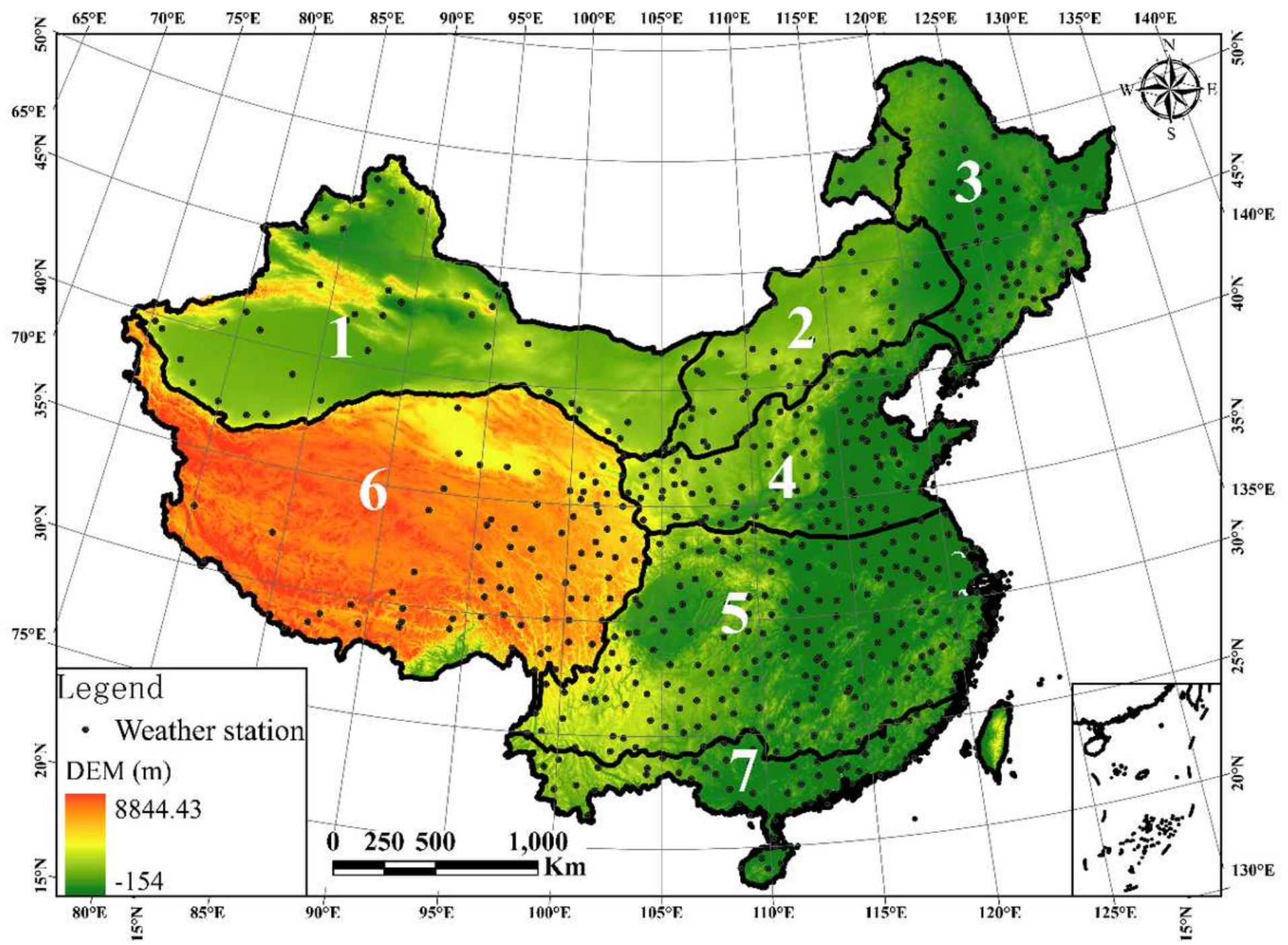

Figure 1

Geological information, DEM, station distribution and sub-region division in China. The numbers in the map denotes different sub-regions. Note: The designations employed and the presentation of the material on this map do not imply the expression of any opinion whatsoever on the part of Research Square concerning the legal status of any country, territory, city or area or of its authorities, or concerning the delimitation of its frontiers or boundaries. This map has been provided by the authors. 


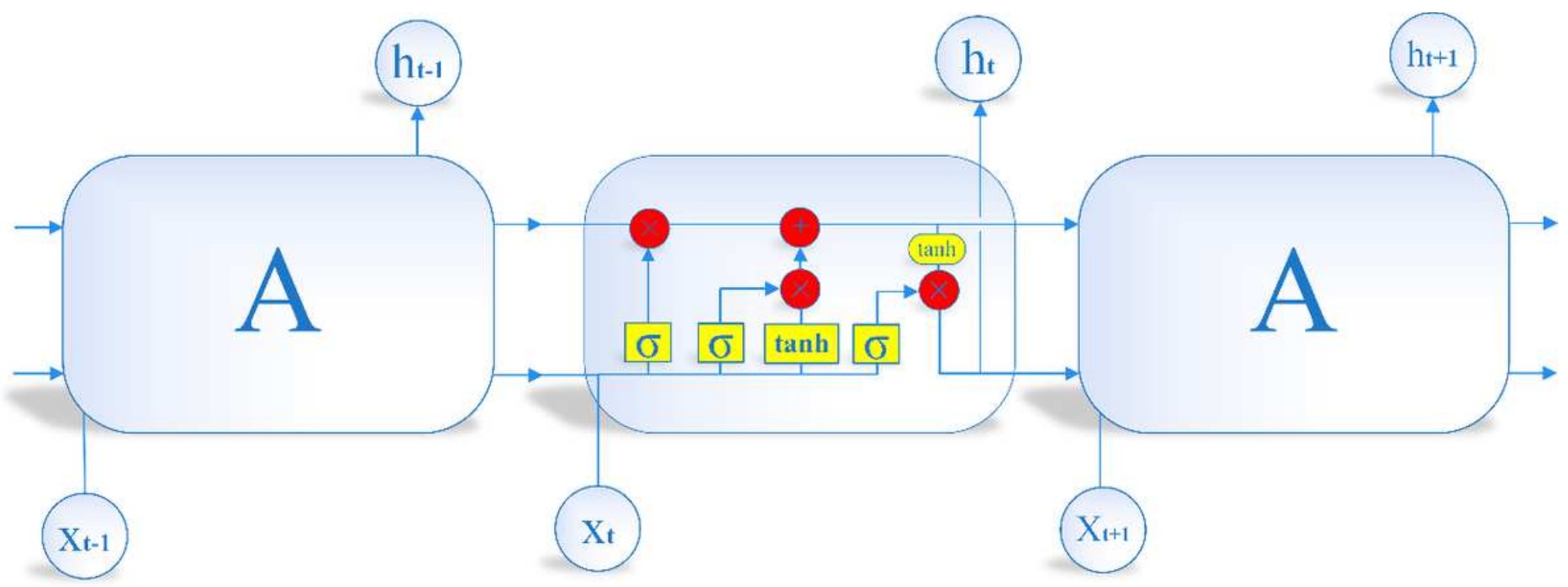

Figure 2

Four interaction layers of the LSTM module chain.

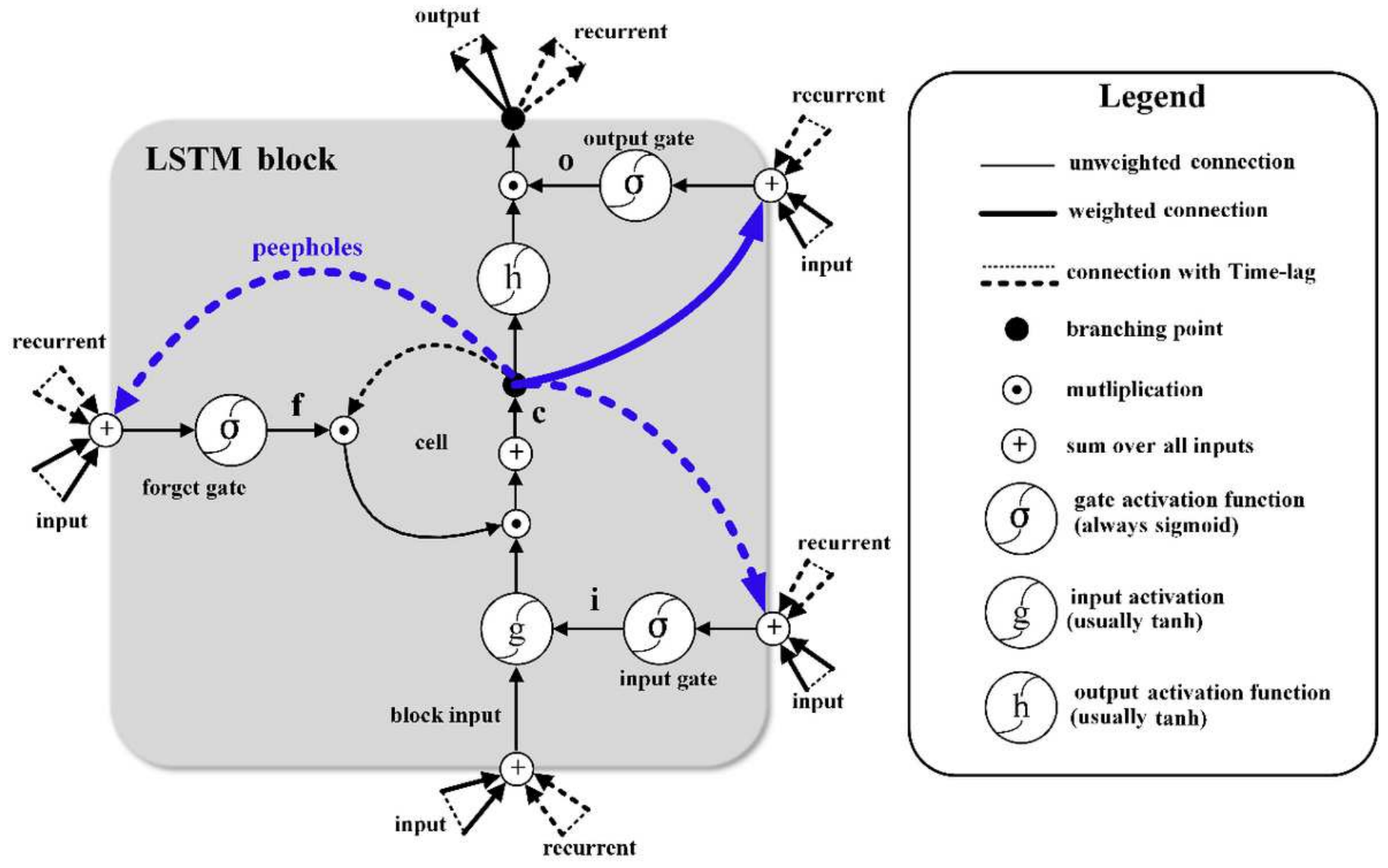

Figure 3

LSTM cell structure in hidden layer. 


\section{Hybrid ARIMA-LSTM Model}

- Data Processing

The six time seale SPI and

SPEI values $(1,3,6,9,12,24)$

of 19 meteorological

stations in Henan Province were calculated

\section{- Forecasting}

Data from 1951-2003 were selected as the training set and data from 2004-2017 were selected as the test set to predict SPI values at four time scales of 19 meteorological stations in Henan Province

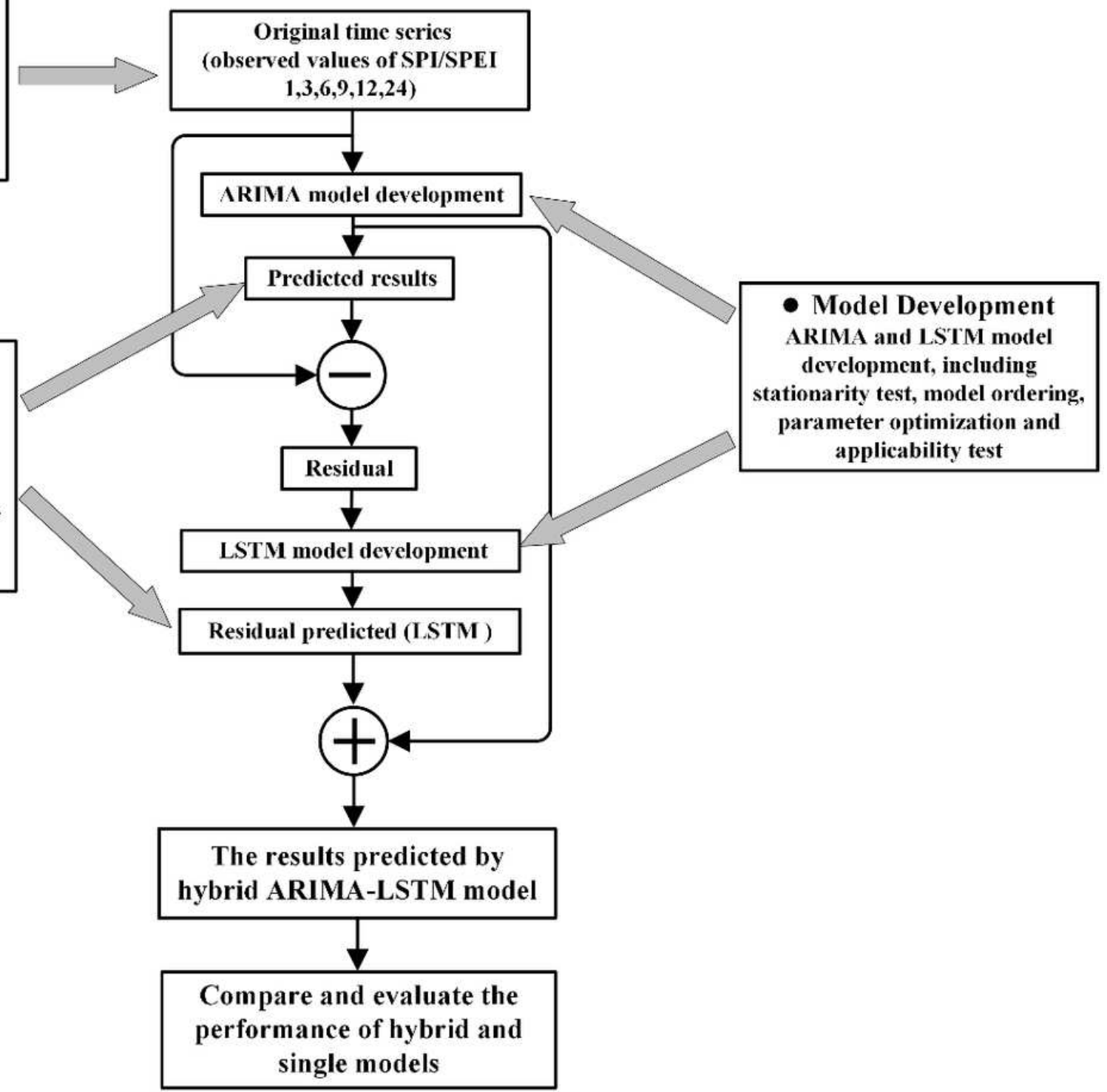

Figure 4

hybrid ARIMA-LSTM model flow chart. 


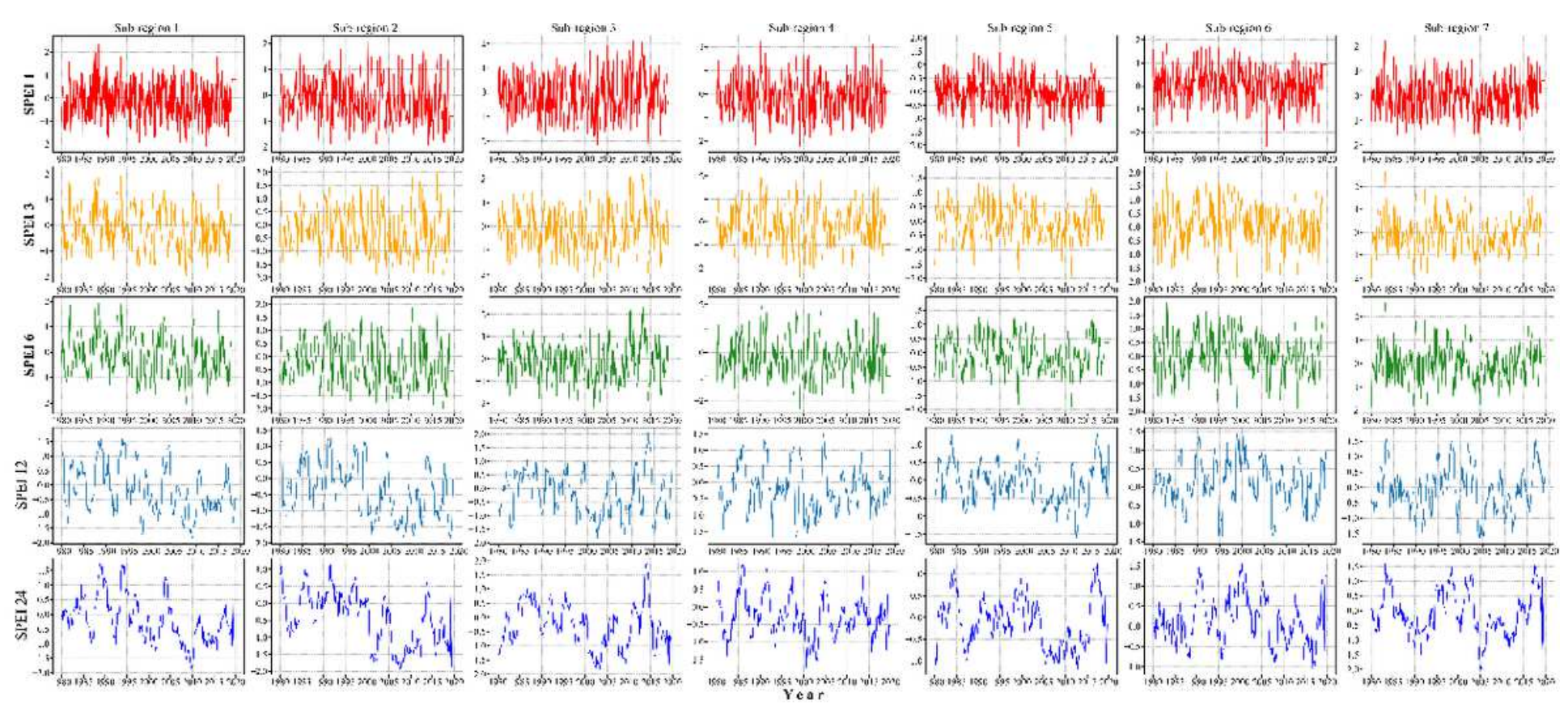

\section{Figure 5}

The variations of SPEl at the 1-, 3-, 6-, 12- and 24-month timescales over 1980-2013 in 7 sub-regions of China.
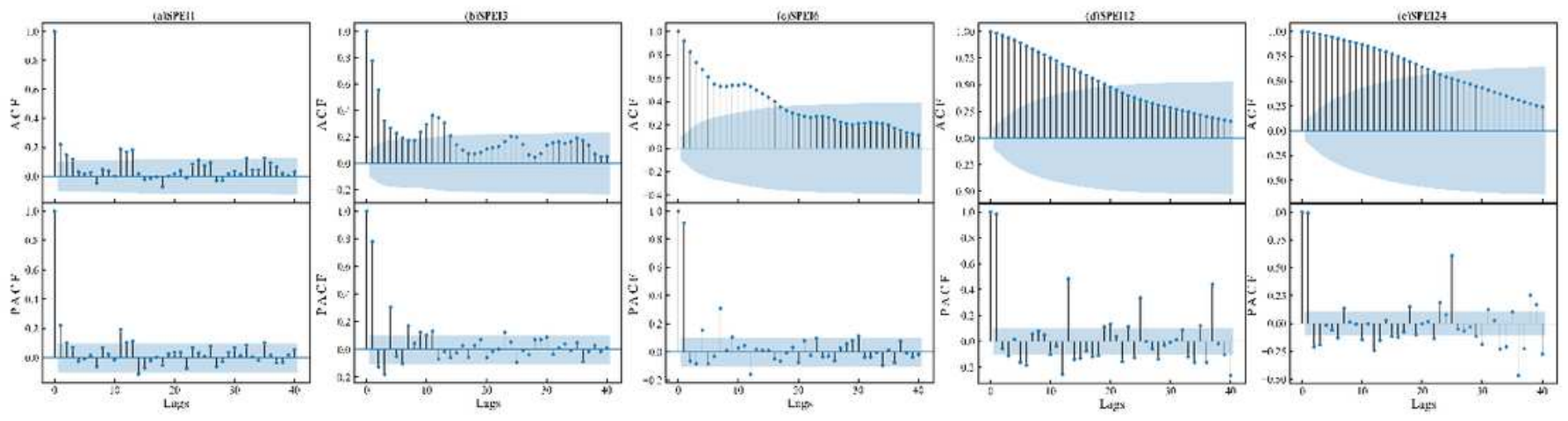

\section{Figure 6}

ACF and PACF of SPEl at the 1-, 3-, 6-, 12- and 24-month timescales in sub-region 1 of China. 

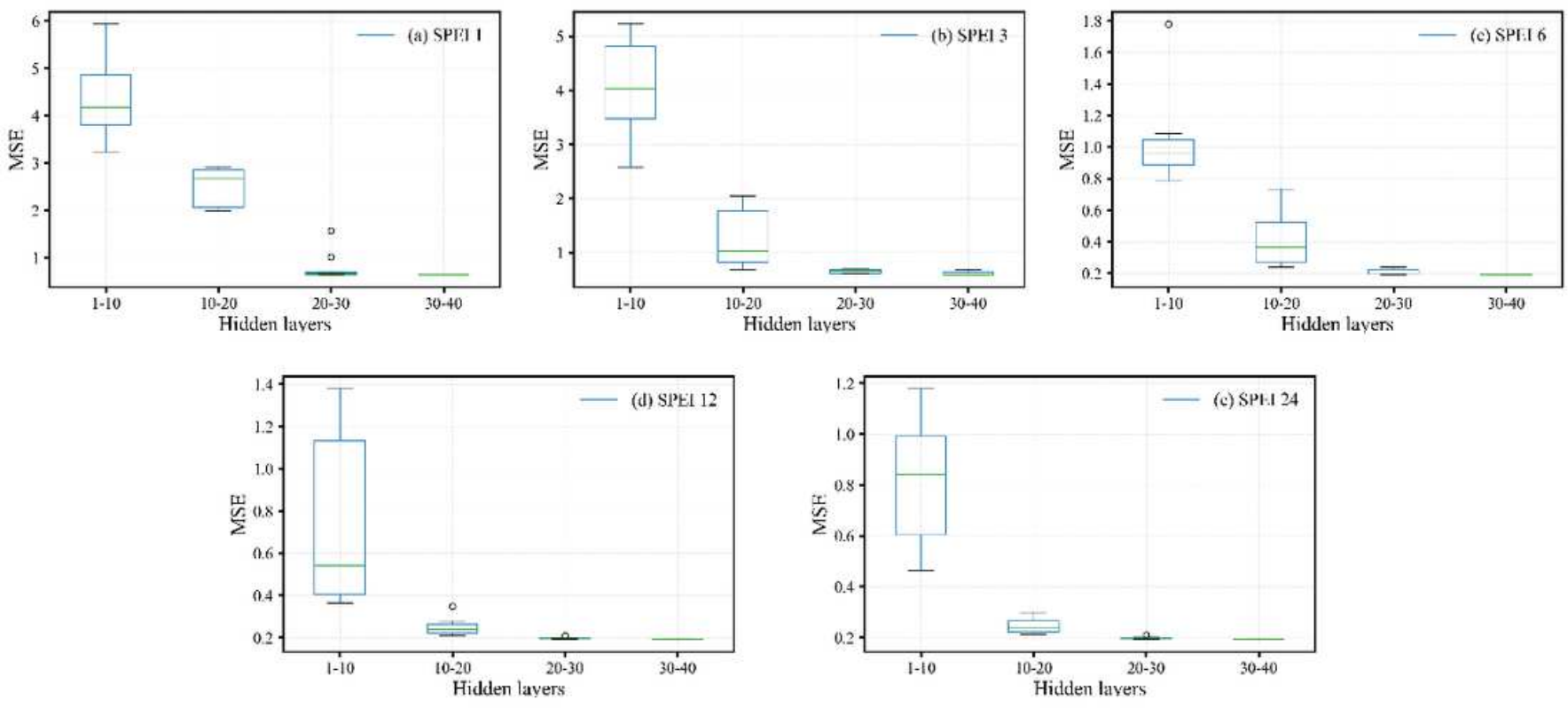

\section{Figure 7}

Multitime scale boxplot of loss fuction and hidden layers of SPEI in sub-region 1 of China. 

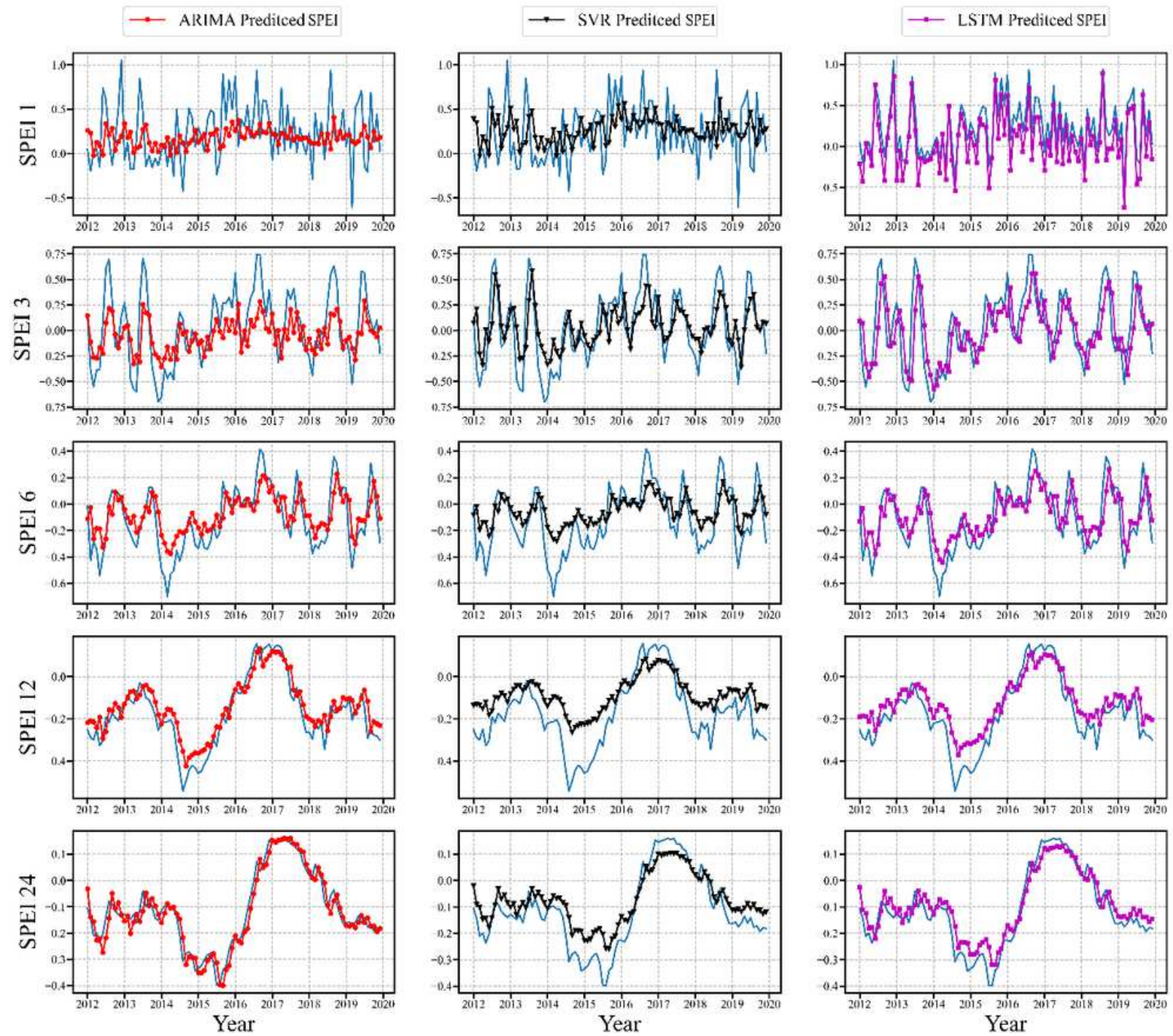

Figure 8

Forecast of multitime scale SPEI value of ARIMA model, SVR model and LSTM model. 

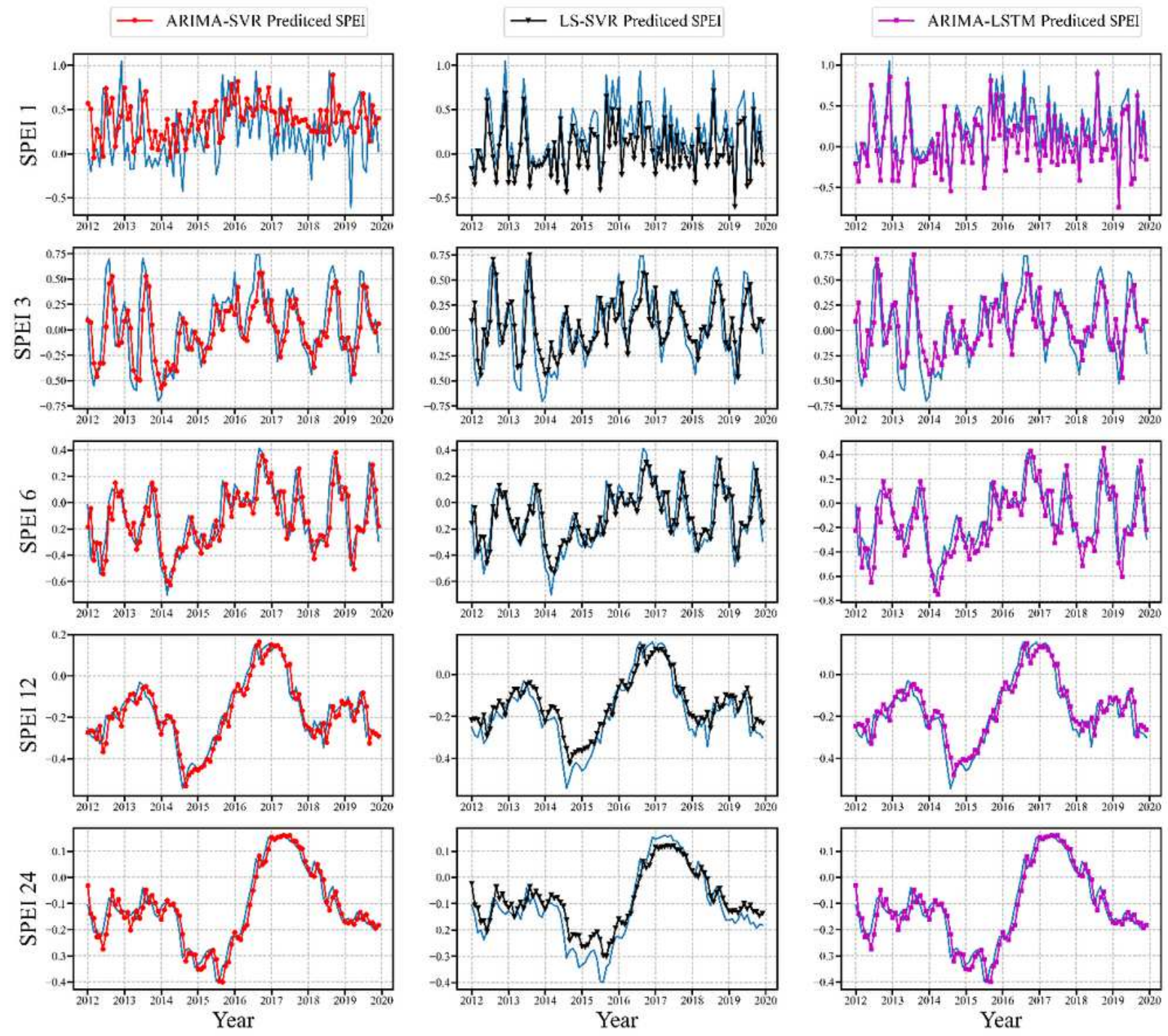

Figure 9

Forecast of multitime scale SPEI value of hybrid ARIMA-SVR model, LS-SVR model and ARIMA-LSTM model. 


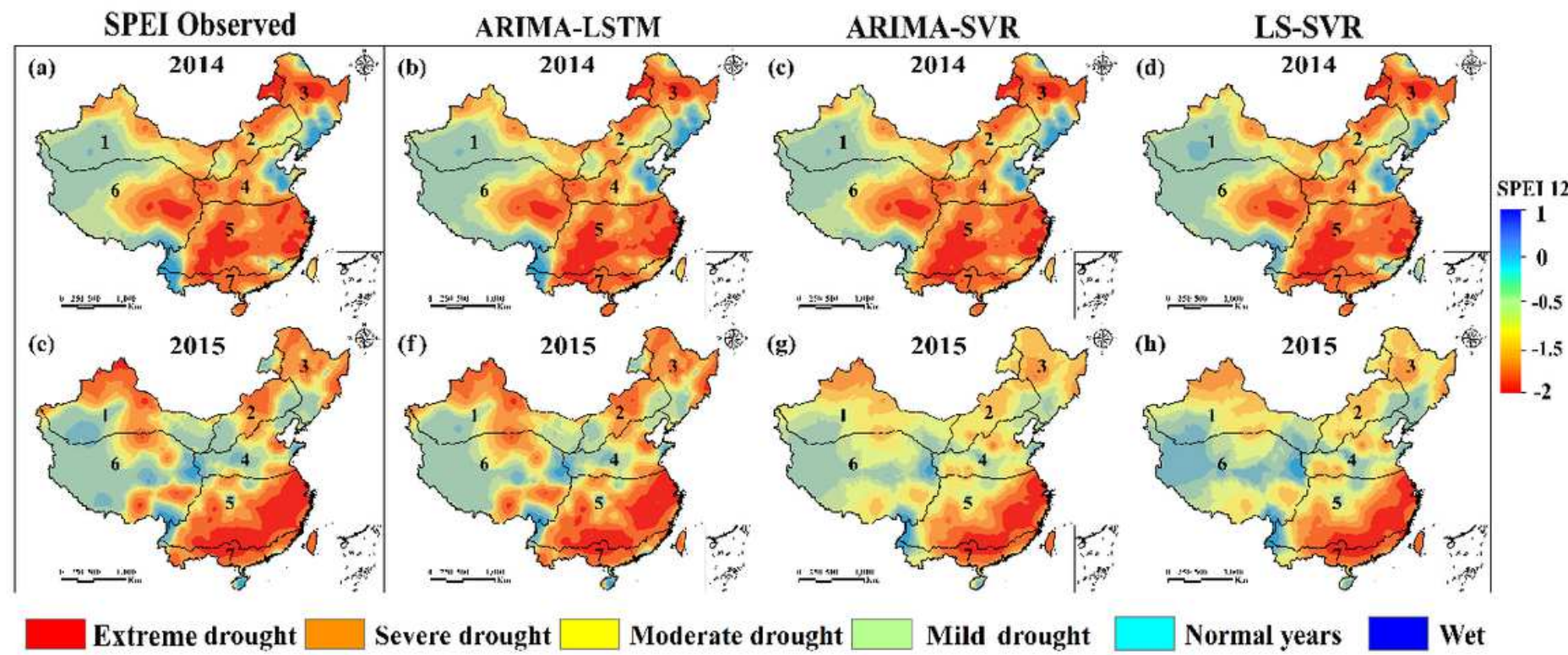

Figure 10

Spatial distributions of SPEI drought levels in 2014 and 2015, using the three forecasting models (ARIMASVR, LS-SVR and ARIMA-LSTM model). Note: The designations employed and the presentation of the material on this map do not imply the expression of any opinion whatsoever on the part of Research Square concerning the legal status of any country, territory, city or area or of its authorities, or concerning the delimitation of its frontiers or boundaries. This map has been provided by the authors. 